\title{
Constraining the Orbit and Mass of epsilon Eridani b with Radial Velocities, Hipparcos IAD-Gaia DR2 Astrometry, and Multiepoch Vortex Coronagraphy Upper Limits
}

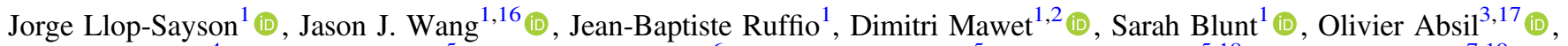
Charlotte Bond ${ }^{4}$, Casey Brinkman ${ }^{5}$, Brendan P. Bowler ${ }^{6}\left({ }_{0}\right.$, Michael Bottom ${ }^{5}$, Ashley Chontos ${ }^{5,18}$ () , Paul A. Dalba ${ }^{7,19}$ (i),

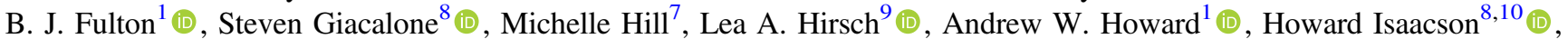
Mikael Karlsson ${ }^{11}$ (10, Jack Lubin ${ }^{12}$ (1), Alex Madurowicz ${ }^{9}$, Keith Matthews ${ }^{1}$, Evan Morris ${ }^{13}$, Marshall Perrin ${ }^{14}$ (i), Bin Ren ${ }^{1}$ (D),

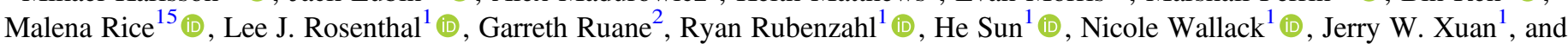
Marie Ygouf $^{2}$

${ }^{1}$ California Institute of Technology, 1200 E California Boulevard, Pasadena, CA 91125, USA; jllopsay@caltech.edu

2 Jet Propulsion Laboratory, California Institute of Technology, 4800 Oak Grove Drive, Pasadena, CA 91109, USA

${ }^{3}$ Space sciences, Technologies \& Astrophysics Research (STAR) Institute Université de Liège (ULiège) 19c allée du Six Août, B-4000 Liège, Belgium

${ }^{4}$ Science and Technology Facilities Council (STFC), Polaris House, North Star Avenue, Swindon, SN2 1SZ, UK

${ }^{5}$ Institute for Astronomy, University of Hawai'i, 2680 Woodlawn Drive, Honolulu, HI 96822, USA

${ }^{6}$ Department of Astronomy, The University of Texas at Austin, Austin, TX 78712, USA

${ }^{7}$ Department of Earth and Planetary Sciences, University of California Riverside, 900 University Avenue, Riverside, CA 92521, USA

501 Campbell Hall, University of California at Berkeley, Berkeley, CA 94720, USA

${ }^{9}$ Kavli Institute for Particle Astrophysics and Cosmology, Stanford University, Stanford, CA 94305, USA

${ }^{10}$ Centre for Astrophysics, University of Southern Queensland, Toowoomba, QLD, Australia

${ }^{11}$ Department of Materials Science and Engineering, Angström Laboratory, Uppsala University, Uppsala, Sweden

${ }^{12}$ Department of Physics \& Astronomy, University of California Irvine, Irvine, CA 92697, USA

${ }^{13}$ Department of Astronomy \& Astrophysics, University of California, Santa Cruz, CA 95064, USA

${ }^{14}$ Space Telescope Science Institute, 3700 San Martin Drive Baltimore, MD 21218, USA

${ }^{15}$ Department of Astronomy, Yale University, New Haven, CT 06511, USA

Received 2021 May 11; revised 2021 June 22; accepted 2021 July 9; published 2021 October 6

\begin{abstract}
$\epsilon$ Eridani is a young planetary system hosting a complex multibelt debris disk and a confirmed Jupiter-like planet orbiting at 3.48 au from its host star. Its age and architecture are thus reminiscent of the early Solar System. The most recent study of Mawet et al., which combined radial-velocity data and Ms-band direct imaging upper limits, started to constrain the planet's orbital parameters and mass, but are still affected by large error bars and degeneracies. Here we make use of the most recent data compilation from three different techniques to further refine $\epsilon$ Eridani b's properties: RVs, absolute astrometry measurements from the Hipparcos and Gaia missions, and new Keck/NIRC2 Ms-band vortex coronagraph images. We combine this data in a Bayesian framework. We find a new mass, $M_{b}=0.66_{-0.09}^{+0.12} \mathrm{M}_{\mathrm{Jup}}$, and inclination, $i=78.81_{-22.41}^{+29.34}$, with at least a factor 2 of improvement over previous uncertainties. We also report updated constraints on the longitude of the ascending node, the argument of the periastron, and the time of periastron passage. With these updated parameters, we can better predict the position of the planet at any past and future epoch, which can greatly help define the strategy and planning of future observations and with subsequent data analysis. In particular, these results can assist the search for a direct detection with JWST and the Nancy Grace Roman Space Telescope's coronagraph instrument.
\end{abstract}

Unified Astronomy Thesaurus concepts: Exoplanet astronomy (486); Exoplanets (498); Radial velocity (1332); Astrometry (80); Direct imaging (387); Debris disks (363)

\section{Introduction}

$\epsilon$ Eridani is a nearby $\mathrm{K} 2 \mathrm{~V}$ dwarf star (Table 1) surrounded by a prominent multibelt debris disk and a confirmed Jupiter-like planet on a $7.4 \mathrm{yr}$ orbit (Mawet et al. 2019). Its relatively young age, spectral type, and architecture are reminiscent of the early Solar System. Its proximity $(3.2 \mathrm{pc})$ and thus apparent brightness $(V=3.73 \mathrm{mag})$ make it an excellent laboratory to study the early formation and evolution of planetary systems analogous to the Solar System.

Since the discovery of its debris disk by the Infrared Astronomical Satellite (Aumann 1985), $\epsilon$ Eridani has been the subject of increased scrutiny culminating with the discovery of

\footnotetext{
1651 Pegasi b Fellow.

${ }_{17}^{17}$ F.R.S.-FNRS Research Associate.

18 NSF Graduate Research Fellow.

19 NSF Astronomy and Astrophysics Postdoctoral Fellow.
}

decade-long radial-velocity (RV) variations by Hatzes et al. (2000) pointing toward the presence of $\mathrm{a} \simeq 1.5 \mathrm{M}_{J}$ giant planet with a period $P=6.9 \mathrm{yr}(\simeq 3$ au orbit) and a high eccentricity $(e=0.6)$. This early orbital solution was dynamically incompatible with the multibelt disk configuration of $\epsilon$ Eridani, raising the possibility of false alarm or at the very least confusion due to stellar jitter. Benedict et al. (2006) attempted at modeling the perturbation caused by the companion using RV combined with HST astrometry. Recently, Mawet et al. (2019) revisited this poster-child system by combining three decades of RV measurements with the most sensitive direct imaging data set ever obtained. The RV data, its state-of-the-art treatment of stellar noise, and direct imaging upper limits were combined in an innovative joint Bayesian analysis, providing new constraints on the mass and orbital parameters of the elusive planet. Mawet et al. (2019) reported a mass of $0.78_{-0.12}^{+0.38}$ $M_{\text {Jup }}$, semimajor axis (SMA) of $3.48 \pm 0.02$ au with a period of 
Table 1

Properties of $\epsilon$ Eridani

\begin{tabular}{lcc}
\hline \hline Property & Value & Ref. \\
\hline R.A. (hms) & $033255.8(\mathrm{~J} 2000)$ & van Leeuwen (2007) \\
Decl. (dms) & $-092729.7(\mathrm{~J} 2000)$ & van Leeuwen (2007) \\
Spectral type & K2V & Keenan \& McNeil (1989) \\
Mass $\left(M_{\odot}\right)$ & $0.82 \pm 0.02$ & Baines \& Armstrong (2011) \\
Distance (pc) & $3.216 \pm 0.0015$ & van Leeuwen (2007) \\
$V$ mag & 3.73 & Ducati (2002) \\
$K$ mag & 1.67 & Ducati (2002) \\
$L$ mag & 1.60 & Cox (2000) \\
$M$ mag & 1.69 & Cox (2000) \\
Age $(\mathrm{Myr})$ & $400-800$ & Mamajek \& Hillenbrand (2008); \\
& & Janson et al. (2015) \\
\hline
\end{tabular}

$7.37 \pm 0.07 \mathrm{yr}$, and an eccentricity of $0.07_{-0.05}^{+0.06}$, an order of magnitude smaller than earlier estimates and consistent with a circular orbit. These new orbital parameters were found to be dynamically compatible to the most recent picture of $\epsilon$ Eridani's disk architecture (Booth et al. 2017; Su et al. 2017; Ertel et al. 2018). However, the joint RV-direct imaging upper limit analysis of Mawet et al. (2019) left some orbital parameters such as inclination $i$, longitude of ascending node $\Omega$, and argument of periapsis $\omega$, largely unconstrained, making future pointed direct detection attempts (e.g., with James Webb Space Telescope; JWST) more difficult.

In this paper, we compiled data from recent Gaia data release 2 (DR2) forming a $25 \mathrm{yr}$ time baseline with archival Hipparcos astrometric data, plus new RV and deep direct imaging observations, to perform a joint astrometry-RV-direct imaging analysis aimed at refining the orbital elements of $\epsilon$ Eridani b. The paper is organized as follows. In Section 2 we present the observations; namely, we include absolute astrometry measurements of $\epsilon$ Eridani to help constrain the orbital parameters and mass. We include an updated set of RV measurements, and new direct imaging observations with Keck/NIRC2. Section 3 describes the methods used to constrain the orbit and mass of $\epsilon$ Eridani by combining the three different observation techniques. At the end of this section, the new constraints are presented. In Section 4 we discuss the findings, update the planet-disk interaction parameters with respect to Mawet et al. (2019) and discuss the prospects of a detection with JWST.

\section{Observations}

\subsection{Radial Velocities}

$\epsilon$ Eridani has been targeted by five multiyear RV planet searches over the past $30 \mathrm{yr}$. Most of the resulting RV measurements are presented and discussed in Mawet et al. (2019). Since the publication of that paper, we have obtained 76 additional spectra of $\epsilon$ Eridani with Keck/HIRES (Vogt et al. 1994), and 172 spectra with the Levy Spectrograph on the Automated Planet Finder (APF) telescope (Radovan et al. 2014; Vogt et al. 2014). These new measurements are given in Table 5. As in Mawet et al. (2019), new HIRES observations were taken using the standard iodine cell configuration used for the California Planet Survey (Howard et al. 2010). Observations were taken through either the B5 $(0$ " $87 \times 3$ ". 5$)$ or $\mathrm{C} 2$ $(0$ ". $87 \times 14$ ". 0$)$ decker, yielding $\mathrm{R} \simeq 55,000$ spectral resolution. Spectra had a median of 293,000 counts per exposure, corresponding to a median extracted flux of 67,000 counts $\mathrm{S} / \mathrm{N}=260$ ) at $550 \mathrm{~nm}$. The same template spectrum described in Mawet et al. (2019), taken in 2010 using the B3 decker, was used to derive RVs from all new spectra obtained for this work.

New APF measurements were also taken using the standard configuration described in Mawet et al. (2019). Most APF measurements were acquired through the $\mathrm{W}$ decker $\left(1^{\prime \prime} \times 3\right.$ "), with a spectral resolution of $\mathrm{R} \simeq 110,000$. The median exposure time across observations was $26 \mathrm{~s}$, yielding a perpixel SNR of 120 at $550 \mathrm{~nm}$. The same template spectrum described in Mawet et al. (2019), taken in 2014 using the $\mathrm{N}$ decker, was used to derive RVs from all new spectra obtained for this work. For our analysis, all exposures taken with a single instrument within $10 \mathrm{hr}$ were binned.

\subsection{Absolute Astrometry Data}

We obtain the astrometry data for $\epsilon$ Eridani from the Hipparcos catalog Hip2 (van Leeuwen 2007), and the Gaia DR2 catalog (Gaia Collaboration et al. 2018), as well as the intermediate astrometry data (IAD) available from the Hipparcos mission.

\subsubsection{Hipparcos Intermediate Astrometry Data}

The Hipparcos data is generally used in its reduced form, consisting of a five-element parameter set: the R.A. $\alpha$, decl. $\delta$, the proper motion (PM) vector $\mu_{\alpha}$ and $\mu_{\delta}$, and the parallax. However, the IAD contains all individual measurements taken by the mission, in the case of $\epsilon$ Eridani, from 1990.036 to 1992.563. The orbit period of $\epsilon$ Eridani b being $\sim 7.31 \mathrm{yr}$, Hipparcos IAD baseline is $\sim 0.35$ times that period. Therefore, Hipparcos IAD may include changes in astrometry that contain signs of the presence of a companion. Indeed, a companion perturbs the otherwise constant rectilinear motion of the photocenter into a Keplerian motion around the barycenter. By using the IAD, we fit our model orbit to the curve described by the measured positions of $\epsilon$ Eridani's photocenter during the Hipparcos mission.

The Hipparcos mission performed its measurements on a 1D scan, the acquisition protocol of which allowed for a reconstruction of the $1 \mathrm{D}$ points into $2 \mathrm{D}$ positions on the sky. The orbital direction of the scan, along with the precise epoch of each measurement are recorded in order to retrieve the final position. We use data from van Leeuwen (2007) that consists of the IAD residuals from the fit to the data (van Leeuwen 2007), the scan direction, epochs, and the errors associated with the original data. Using the fit from van Leeuwen (2007) and the residuals combined with the scan direction data, we reconstruct the one-dimensional scan measurements following the method used in Nielsen et al. (2020). The available data consists of 78 data points; Table 6 lists the complete set of data points used.

\subsubsection{Gaia DR2}

Unlike with Hipparcos, the IAD for Gaia is not yet public. We thus utilize the reduced data consisting of five astrometric parameters: the R.A. $\alpha_{G}$, decl. $\delta_{G}$, the PM vectors $\mu_{\alpha, G}$ and $\mu_{\delta, G}$, and the parallax. Although the velocity in the data is recorded as a PM, in reality, in the presence of a companion, this velocity contains both the PM and the velocity induced by the movement of the photocenter around the barycenter of the system. The deviation of the velocity from the actual PM is often called the PM anomaly, which stems from the movement of the photocenter around the barycenter. 
Table 2

Summary of Observations of $\epsilon$ Eridani with Keck/NIRC2 in Ms Band

\begin{tabular}{|c|c|c|c|c|c|c|c|c|}
\hline Epoch & $\begin{array}{l}\text { Tot. time } \\
\text { (s) }\end{array}$ & $\begin{array}{l}\text { Exp. time } \\
\text { (s) }\end{array}$ & Coadds & \# of exp. & $\begin{array}{l}\text { Angle rot. } \\
\text { (deg) }\end{array}$ & $\begin{array}{c}5 \sigma \\
0 ! 4\end{array}$ & $\begin{array}{c}5 \sigma \\
1 ! 0\end{array}$ & WFS \\
\hline 2017-01-09 & 6300 & 0.5 & 60 & 210 & 88.6 & $4.8 \mathrm{e}-05$ & $1.1 \mathrm{e}-05$ & $\mathrm{SH}$ \\
\hline 2017-01-10 & 7800 & 0.5 & 60 & 260 & 100.2 & $4.9 \mathrm{e}-05$ & $1.1 \mathrm{e}-05$ & $\mathrm{SH}$ \\
\hline 2017-01-11 & 4800 & 0.5 & 60 & 160 & 69.5 & $6.3 e-05$ & $1.0 \mathrm{e}-05$ & $\mathrm{SH}$ \\
\hline Combined 2017 & $5.25 \mathrm{~h}$ & & & & & $3.3 e-05$ & $7.0 \mathrm{e}-06$ & \\
\hline 2019-10-20 & 5190 & 0.25 & 120 & 173 & 84.9 & $5.1 \mathrm{e}-05$ & $2.0 \mathrm{e}-05$ & Pyramid \\
\hline 2019-10-21 & 5850 & 0.25 & 120 & 195 & 82.0 & $3.9 \mathrm{e}-05$ & $1.8 \mathrm{e}-05$ & Pyramid \\
\hline 2019-10-22 & 7170 & 0.25 & 120 & 239 & 89.3 & $5.4 \mathrm{e}-05$ & $2.0 \mathrm{e}-05$ & Pyramid \\
\hline 2019-11-04 & 6660 & 0.25 & 120 & 222 & 68.2 & $6.3 e-05$ & $1.8 \mathrm{e}-05$ & Pyramid \\
\hline 2019-11-05 & 6510 & 0.25 & 120 & 217 & 68.0 & $4.6 e-05$ & $1.3 \mathrm{e}-05$ & Pyramid \\
\hline 2019-12-08 & 6510 & 0.3 & 100 & 217 & 103.7 & $1.0 \mathrm{e}-04$ & $2.4 \mathrm{e}-05$ & SH \\
\hline Combined 2019 & $10.5 \mathrm{~h}$ & & & & & $2.3 e-05$ & $7.9 \mathrm{e}-06$ & \\
\hline
\end{tabular}

Note. From left to right: UT date of the observation, total exposure time, exposure time per integration, number of coadds, number of exposures, total paralactic angle rotation for angular differential imaging, $5 \sigma$ sensitivity expressed as the planet-to-star flux ratio at 2 projected separation $(0$ ". 4 and 1 ". 0 ), and finally the wave front sensor (WFS) used (Shack-Hartmann or Pyramid).

Due to $\epsilon$ Eridani's proximity and the long baseline between Hipparcos and Gaia, we need to account for the 3D effects of different tangential planes where the PMs of each data set are published. In other words, we account for the effect of the curvature when comparing the two coordinate systems. Correcting for this effect when propagating from the Hipparcos' to Gaia's epoch accounts for an error of $\sim 0.25$ mas for the PM in the RA direction, which, although being below one sigma, is large enough to affect the model fit. We use the SkyCoord tool in the astropy Python package to propagate between epochs that accounts for the difference in epochs and position in spherical coordinates. This allows us to work combining velocities from both data sets. We follow a similar approach to Kervella et al. (2019).

Furthermore, we correct for systematics from Gaia's rotating frame of reference according to the method described in Lindegren et al. (2018).

\subsubsection{Gaia eDR3}

We use the Gaia eDR3 to do a different fit to the PM anomalies between the Hipparcos and Gaia PMs. We use the calibrated PMs from Brandt (2021). This catalog includes the PMs from the Hipparcos epoch, from the Gaia eDR3 epoch, and the long-term PM computed with the positions at the two epochs and the time difference. The difference between the Hipparcos or Gaia PM and the long baseline PM is the PM anomaly, which is caused by the presence of the planet. For this fit we do not use the Hipparcos IAD.

\subsection{Direct Imaging Data}

Building upon the work presented by Mawet et al. 2019, we present additional Ms-band observations on the $\epsilon$ Eridani system acquired in 2019 with Keck/NIRC2 and its vector vortex coronagraph (Serabyn et al. 2017). The observations are summarized in Table 2. Except for the night of the 2019 December 08, the new data was acquired using the newly installed infrared pyramid wave front sensor (Bond et al. 2020) instead of the facility Shack-Hartmann wave front sensor. The alignment of the star behind the coronagraph is ensured at the 2 mas level by the quadrant analysis of coronagraphic images for tip-tilt sensing (Huby et al. 2015, 2017). The telescope was used in pupil tracking mode to allow speckle subtraction with angular differential imaging (Marois et al. 2008).

The data was corrected for bad pixels, flat-fielded, skysubtracted (accounting for detector illumination), and registered following the method detailed in Xuan et al. (2018). Similar to Mawet et al. (2019), the stellar point-spread function (PSF) was subtracted using principal component analysis (PCA) combined with a matched filter (Ruffio et al. 2017) from the opensource Python package pyKLIP (Wang et al. 2015). After highpass filtering the images with a Gaussian filter with a length scale of twice the PSF full width at half maximum (FWHM), the PSF FWHM is $\sim 10$ pixels $\left(\sim 0 !^{\prime \prime} 1\right)$ in Ms band. Stellar speckles are subtracted using 30 principal components. For each science frame, the principal components are only calculated from images featuring an azimuthal displacement of the planet on the detector of more than 0.5 FWHM. The final sensitivity of each epoch is plotted in Figure 1 as a function of projected separation. The nightly performance in 2019 was lower than in 2017 resulting in a similar combined sensitivity for each year despite the longer combined exposure time. A weighted mean is used to combine different epochs together. As a way to correct for any correlation between frames, the noise of the combined images is then normalized following the standard procedure of dividing by the standard deviation calculated in concentric annuli. With the exception of the 2019 December 08 observation, the epochs in 2017 and 2019 are close enough in time for the planet to not move significantly compared to the PSF size with a full width at half maximum of $\sim 10$ pixels.

\section{Analysis \\ 3.1. Radial-velocity Model}

Mawet et al. (2019) performed a thorough series of tests to evaluate the possibility that $\epsilon$ Eridani $\mathrm{b}$ is an artifact of stellar activity, finding that the $\sim 7 \mathrm{yr}$ orbital period is distinct from periods and harmonics of the periodicities in the $\mathrm{S}_{\mathrm{HK}}$ activity indicator timeseries. Our aim is not to recapitulate their analysis, but to update their orbital solution using the additional data obtained since the paper's publication, which spans approximately half of one orbital period of $\epsilon$ Eridani $b$. 

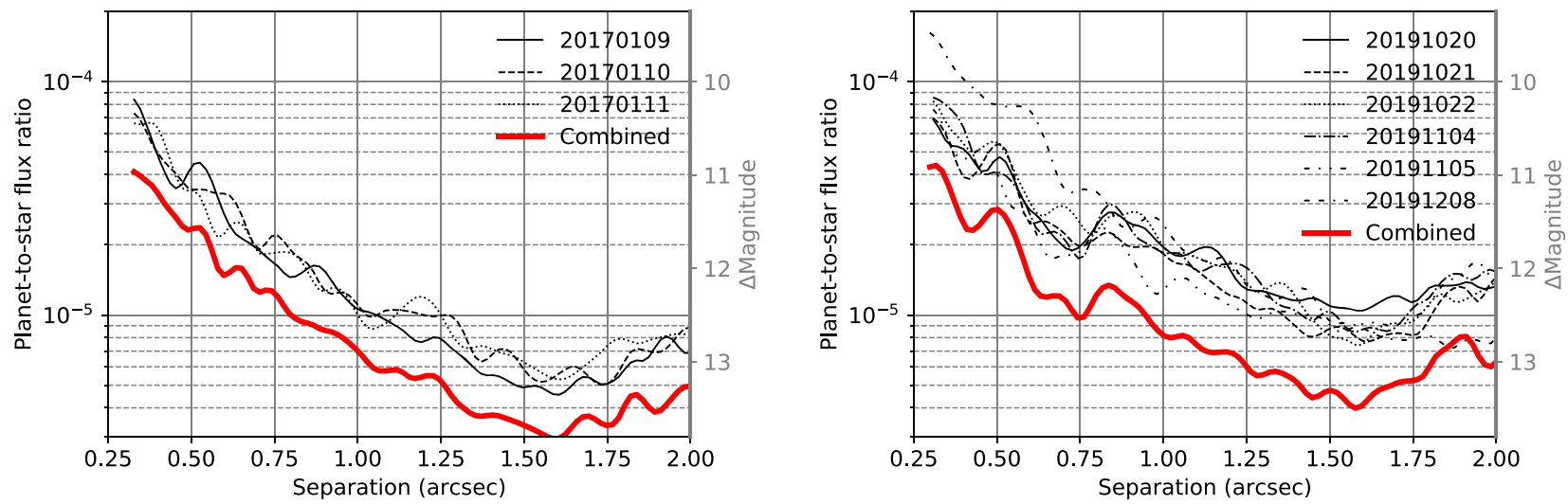

Figure 1. Planet sensitivity for each observations in 2017 (left) and 2019 (right). The planet sensitivity is expressed as the $5 \sigma$ planet-to-star flux ratio. The 2019 December 8 epoch was not included in the combined sensitivity curve for 2019 as the planet would have moved by an amount comparable to the size of the PSF.

Mawet et al. (2019) identified three peaks in a Lomb-Scargle periodogram of the RVs that rose above the $1 \%$ eFAP: one at the putative planet period of $7.3 \mathrm{yr}$, one at $2.9 \mathrm{yr}$, and one at 11 day. Applying RVSearch (Rosenthal et al. 2021) to the full data set, we recover this structure of peaks. Like Mawet et al. (2019), we also identify two major periods in the $S_{\mathrm{HK}}$ time series for both HIRES and APF, which coincide with the 11 day and $2.9 \mathrm{yr}$ periods in the RVs. We interpret these as the signatures of rotationally-modulated stellar activity and a longterm activity cycle, respectively. To investigate the effects of these signals on the physical parameters of planet $b$, we performed two separate RV-orbit fits using RadVel (Fulton et al. 2018) to try different priors on the Gaussian-process (GP) timescale for modeling the stellar activity. In each of these fits, we assume a one-planet orbital solution, parameterized as $\sqrt{e_{b}} \cos \omega_{b}, \sqrt{e_{b}} \sin \omega_{b}, \mathrm{~T}_{\text {conj,b }}, \mathrm{P}_{b}, \mathrm{~K}_{b}$. We also included RV offset $(\gamma)$ and white noise $(\sigma)$ parameters for each instrument in the fit, treating the four Lick velocity data sets independently to account for instrumental upgrades as in Mawet et al. (2019). Finally, we allowed an RV trend $\dot{\gamma}$.

In the first fit, we included a GP noise model to account for the impact of rotationally-modulated magnetic activity on the RVs (Rajpaul et al. 2015). We used a quasiperiodic kernel, following (Mawet et al. 2019). This kernel has hyperparameters $\eta_{2}$, the exponential decay timescale (analagous to the lifetime of active regions on the stellar disk), $\eta_{3}$, the stellar rotation period, $\eta_{4}$, which controls the number of local maxima in the RVs per rotation period, and $\eta_{1}$, the amplitude of the GP mean function, which we treated as independent for each instrument data set. Following López-Morales et al. (2016), we fixed $\eta_{4}$ to 0.5 , which allows approximately two local maxima per rotation period. In this first fit, we allowed $\eta_{2}$ and $\eta_{3}$ to vary in the range (0 to 100 day). We calculated a Markov-chain representation of the posterior using emcee (Foreman-Mackey et al. 2013), We visually inspected the chains to ensure appropriate burn in and production periods. In total, the chain contained 450,080 samples. The resulting orbital and nuisance parameters are given in Table 3. Both the orbital parameters and the GP hyperparameters are well constrained; in particular, the marginalized posterior over the rotation period $\eta_{3}$ is Gaussian about the expected period of 11 day. The data allow a trend, although the value is consistent with no trend at the $1 \sigma$ level,
Table 3

RV-fit MCMC Posteriors

\begin{tabular}{|c|c|c|c|}
\hline Parameter & Credible Interval & Maximum Likelihood & Units \\
\hline \multicolumn{4}{|c|}{ Modified MCMC Step Parameters } \\
\hline$P_{b}$ & $2671_{-23}^{+17}$ & 2661 & days \\
\hline$T \operatorname{conj}_{b}$ & $2,460,017_{-32}^{+76}$ & 2460023 & JD \\
\hline Tperi $_{b}$ & $2,460,054_{-690}^{+680}$ & 2460235 & JD \\
\hline$e_{b}$ & $0.055_{-0.039}^{+0.067}$ & 0.046 & \\
\hline$\omega_{b}$ & $57.3_{-154.7}^{+80.2}$ & 2.1 & $\circ$ \\
\hline$K_{b}$ & $10.34_{-0.93}^{+0.95}$ & 10.33 & $\mathrm{~m} \mathrm{~s}^{-1}$ \\
\hline \multicolumn{4}{|c|}{ Other Parameters } \\
\hline$\gamma_{\text {lick} 4}$ & $-1.0_{-2.6}^{+2.7}$ & -0.9 & $\mathrm{~m} \mathrm{~s}^{-1}$ \\
\hline$\gamma_{\text {lick3 }}$ & $10.0 \pm 4.9$ & 9.9 & $\mathrm{~m} \mathrm{~s}^{-1}$ \\
\hline$\gamma_{\text {lick2 }}$ & $7.5_{-5.7}^{+5.6}$ & 7.6 & $\mathrm{~m} \mathrm{~s}^{-1}$ \\
\hline$\gamma_{\text {lick1 }}$ & $4.4_{-6.1}^{+6.2}$ & 4.5 & $\mathrm{~m} \mathrm{~s}^{-1}$ \\
\hline$\gamma_{\text {hires }_{j}}$ & $2 \pm 1$ & 2 & $\mathrm{~m} \mathrm{~s}^{-1}$ \\
\hline$\gamma_{\text {harps }}$ & $16,442.5_{-3.1}^{+3.2}$ & $16,442.4$ & $\mathrm{~m} \mathrm{~s}^{-1}$ \\
\hline$\gamma_{\mathrm{ces}}$ & $16,446.6_{-5.5}^{+5.7}$ & $16,446.6$ & $\mathrm{~m} \mathrm{~s}^{-1}$ \\
\hline$\gamma_{\text {apf }}$ & $-0.9 \pm 1.3$ & -0.9 & $\mathrm{~m} \mathrm{~s}^{-1}$ \\
\hline$\dot{\gamma}$ & $-0.00026_{-0.0006}^{+0.00063}$ & -0.00026 & $\mathrm{~m} \mathrm{~s}^{-1} \mathrm{~d}^{-1}$ \\
\hline$\ddot{\gamma}$ & $\equiv 0.0$ & $\equiv 0.0$ & $\mathrm{~m} \mathrm{~s}^{-1} \mathrm{~d}^{-2}$ \\
\hline$\sigma_{\text {lick4 }}$ & $5.17_{-0.95}^{+1.1}$ & 5 & $\mathrm{~m} \mathrm{~s}^{-1}$ \\
\hline$\sigma_{\text {lick3 }}$ & $5.6_{-2.3}^{+2.1}$ & 5.3 & $\mathrm{~m} \mathrm{~s}^{-1}$ \\
\hline$\sigma_{\text {lick2 }}$ & $2.3_{-1.4}^{+3.6}$ & 0.5 & $\mathrm{~m} \mathrm{~s}^{-1}$ \\
\hline$\sigma_{\text {lick1 }}$ & $7.2_{-3.7}^{+3.5}$ & 7.4 & $\mathrm{~m} \mathrm{~s}^{-1}$ \\
\hline$\sigma_{\text {hires } j}$ & $2.36_{-0.41}^{+0.46}$ & 2.26 & $\mathrm{~m} \mathrm{~s}^{-1}$ \\
\hline$\sigma_{\text {harps }}$ & $4.8_{-2.7}^{+2.2}$ & 4.3 & $\mathrm{~m} \mathrm{~s}^{-1}$ \\
\hline$\sigma_{\mathrm{ces}}$ & $8.1_{-4.5}^{+3.8}$ & 7.3 & $\mathrm{~m} \mathrm{~s}^{-1}$ \\
\hline$\sigma_{\mathrm{apf}}$ & $3.64_{-0.55}^{+0.62}$ & 3.51 & $\mathrm{~m} \mathrm{~s}^{-1}$ \\
\hline$\eta_{1, \text { apf }}$ & $7.82_{-0.92}^{+0.97}$ & 7.71 & $\mathrm{~m} \mathrm{~s}^{-1}$ \\
\hline$\eta_{1, \text { hires } j}$ & $6.92_{-0.59}^{+0.64}$ & 6.78 & $\mathrm{~m} \mathrm{~s}^{-1}$ \\
\hline$\eta_{1, \mathrm{ces}}$ & $7.5_{-4.7}^{+4.1}$ & 7.0 & $\mathrm{~m} \mathrm{~s}^{-1}$ \\
\hline$\eta_{1, \text { harps }}$ & $5.7_{-3.0}^{+2.3}$ & 5.3 & $\mathrm{~m} \mathrm{~s}^{-1}$ \\
\hline$\eta_{1, \text { lick,fischer }}$ & $8.7_{-1.4}^{+1.3}$ & 8.0 & \\
\hline$\eta_{2}$ & $37.6_{-5.4}^{+6.4}$ & 36.4 & days \\
\hline$\eta_{3}$ & $11.68_{-0.13}^{+0.14}$ & 11.66 & days \\
\hline$\eta_{4}$ & $\equiv 0.5$ & $\equiv 0.5$ & \\
\hline
\end{tabular}

Note. 436,160 links saved

Reference epoch for $\gamma, \dot{\gamma}, \ddot{\gamma}: 2,457,454.642028$. 
which allows us to conclude that there is no evidence in the current data for a RV trend. One nonintuitive feature of this fit is a preference for extremely small values $\left(10^{-6} \mathrm{~m} \mathrm{~s}^{-1}\right)$ of white noise jitter for the second Lick data set. Even when we ran a fit requiring that all jitter values be at least $0.5 \mathrm{~m} \mathrm{~s}^{-1}$, the posterior peaked at this lower bound. This may be evidence that much of the noise in this particular data set is correlated, and therefore well modeled by the GP noise model. It could also indicate that the reported observational uncertainties are overestimated for this data set. Whatever the reason, there are fewer than 10 measurements that are affected by the value of this jitter parameter, and neither the orbital parameters nor the GP timescale parameters are affected by its particular value.

To investigate the impact of the long-term activity cycle on the marginalized orbital parameter posteriors, we performed another fit identical to the one described above, except we required that $\eta_{2}$ and $\eta_{3}$ vary between $1 \mathrm{yr}$ and the $\sim 30 \mathrm{yr}$ observation baseline. The marginalized posteriors for $\eta_{2}$ and $\eta_{3}$ were broad, with power across the entire allowed space, although the period parameter $\eta_{3}$ showed local maxima at both $\sim 1100$ and $\sim 2000$ day. The marginalized 1 day posteriors for both of these parameters did not vary with those of any of the orbital parameters, allowing us to conclude that the long-term activity cycle, while somewhat present in the RVs, did not significantly affect the derived values of the orbital parameters. We therefore adopt the rotation-only GP fit described above.

Our derived orbital parameters, accounting for the effect of rotationally-modulated stellar noise, are very similar to those of Mawet et al. (2019; see their Table 3). We derive an orbital period of $2671_{-23}^{+17}$ days, a slightly reduced median semiamplitude of $10.3 \mathrm{~m} \mathrm{~s}^{-1}$, and a low median eccentricity of 0.067 . We show the series of RV measurements, the residuals to the fit and the phase-folded RV curve in Figure 2.

\subsection{Combining Direct Imaging, Astrometry, and Radial- velocity Data}

We use the RV posterior distributions presented in Section 3.1 that we obtain from the RadVel orbit fit as the prior probabilities for the model fit to the astrometry and direct imaging data. The set of priors from the RV fit consists of: $a, e$, $\omega, \tau$, and $M \sin i$. We use the same orbital parameter convention as in Blunt et al. (2020). However, since the fit to the astrometry and direct imaging data will obtain a separate distribution for both the mass and inclination, we draw a set of correlated values for $i$ and $M$ from the $M \sin i$ distribution. We assume a sine distribution as a prior probability for $i$, which corresponds to an unconstrained prior for $i$.

Such a set of parameters presents a complex covariance that is practically impossible to reproduce if trying to represent these distributions in a parametric way. Instead, we choose to use a kernel density estimator (KDE) to translate the posteriors to priors. A KDE smooths a probability density by convolving a Gaussian kernel with the discrete points of the Markov-chain Monte Carlo (MCMC). It allows one to preserve the covariance information contained in the original posteriors while allowing for a reliable reproduction of the shapes in the distributions. We use the scipy.stats package implementation of a KDE, which allows for a customized value of the KDE bandwidth parameter. The choice of the bandwidth is critical to maintain the fidelity of the RV fit for the fit of the astrometry and direct imaging data. We explain the process to compute the optimal bandwidth for our data in Appendix C.
The combined astrometric and imaging model consists of thirteen parameters: the six orbital parameters $(a, e, i, \omega, \Omega, \tau)$, the mass of the companion $M_{b}$, the total mass of the system $M_{\text {tot }}$, the parallax, the position of the barycenter of the system at an arbitrary epoch (we choose the Hipparcos epoch at 1992.25, $\alpha_{1992.25}$, and $\delta_{1992.25}$ ), and the PM vector of the barycenter $\mu=\left[\mu_{\alpha}, \mu_{\delta}\right]$.

\subsection{Astrometric Model}

For the astrometric model, we use a similar approach to Rosa et al. (2019a) and Nielsen et al. (2020) while also fitting for the parallax and $M_{\text {tot }}$. This framework consists on deriving from the model a displacement of the photocenter from the barycenter induced by the presence of a companion. The orbital parameters and the relative mass are used to compute this relative motion of the photocenter about the barycenter. Then, to compare to the astrometry data, we obtain absolute astrometry quantities propagating from a reference R.A./decl. position with the PM of the barycenter, and adding the relative displacement. For this reason we add to our model the reference position $\alpha_{1992.25}$ and $\delta_{1992.25}$, and the PM of the barycenter $\mu$. We assume that the brightness of the planet is negligible compared to the host star (below $10 \mathrm{ppb}$ reflected light in the visible); consequently making the photocenter and the star positions coincide.

We compute the goodness of fit to the astrometric data by deriving two distinct terms for both the Hipparcos and Gaia data: $\chi_{\text {astrom }}^{2}=\chi_{H}^{2}+\chi_{G}^{2}$. For the Hipparcos IAD, we calculate the expected position of the barycenter at all IAD epochs $\left(\alpha_{H}\right.$ and $\left.\delta_{H}\right)$ by propagating $\alpha_{1992.25}$ and $\delta_{1992.25}$ with $\mu$. The displacement of the photocenter with respect to the barycenter $\left(\Delta \alpha_{H}\right.$ and $\Delta \delta_{H}$ ) is computed using the expected orbit from the model orbital parameters, and the relative mass. We compare this values to the corresponding $\alpha_{\text {IAD }}$ and $\delta_{\text {IAD }}$ that we calculate from the 1D scans from IAD. We compute $\chi_{H}^{2}$ in the same way as in Nielsen et al. (2020).

Similarly for the Gaia data, we propagate the reference position of the barycenter, $\alpha_{1992.25}$ and $\delta_{1992.25}$, to the Gaia epoch $\alpha_{G}$ and $\delta_{G}$, and we compute the displacement of the photocenter with respect to the barycenter $\left(\Delta \alpha_{G}\right.$ and $\left.\Delta \delta_{G}\right)$ with the orbital parameters and the relative mass. We compare this derived quantity to the Gaia DR2 positional data, i.e., the R.A./decl. from the Gaia DR2 catalog $\alpha_{\mathrm{G}, \mathrm{GDR} 2}$ and $\delta_{\mathrm{G}, \mathrm{GDR} 2}$. We can fit to the Gaia DR2 PM data by adding an instantaneous PM disturbance to the reference PM calculated by deriving an average linear velocity of the star due to its orbital motion over a few epochs around 2015.5. The $\chi_{G}^{2}$ associated with the Gaia data is then calculated in the same way as described in Rosa et al. (2019a).

The analysis of the Hipparcos-Gaia eDR3 acceleration data is done in the way described in Kervella et al. (2019).

\subsection{Direct Imaging Model}

The part of the likelihood function associated with the direct imaging data described in Section 2.3 is computed based on the method described in Mawet et al. (2019). The logarithm of the direct imaging likelihood $(\mathcal{P})$ (Ruffio et al. 2018) for a single epoch can be written as:

$$
\log \mathcal{P}(d \mid I, x)=-\frac{1}{2 \sigma_{x}^{2}}\left(I^{2}-2 I \tilde{I}_{x}\right)
$$

where $I$ is the planet flux corresponding to the planet mass in the orbital model, $\tilde{I}_{x}$ is the estimated flux from the data at the location 

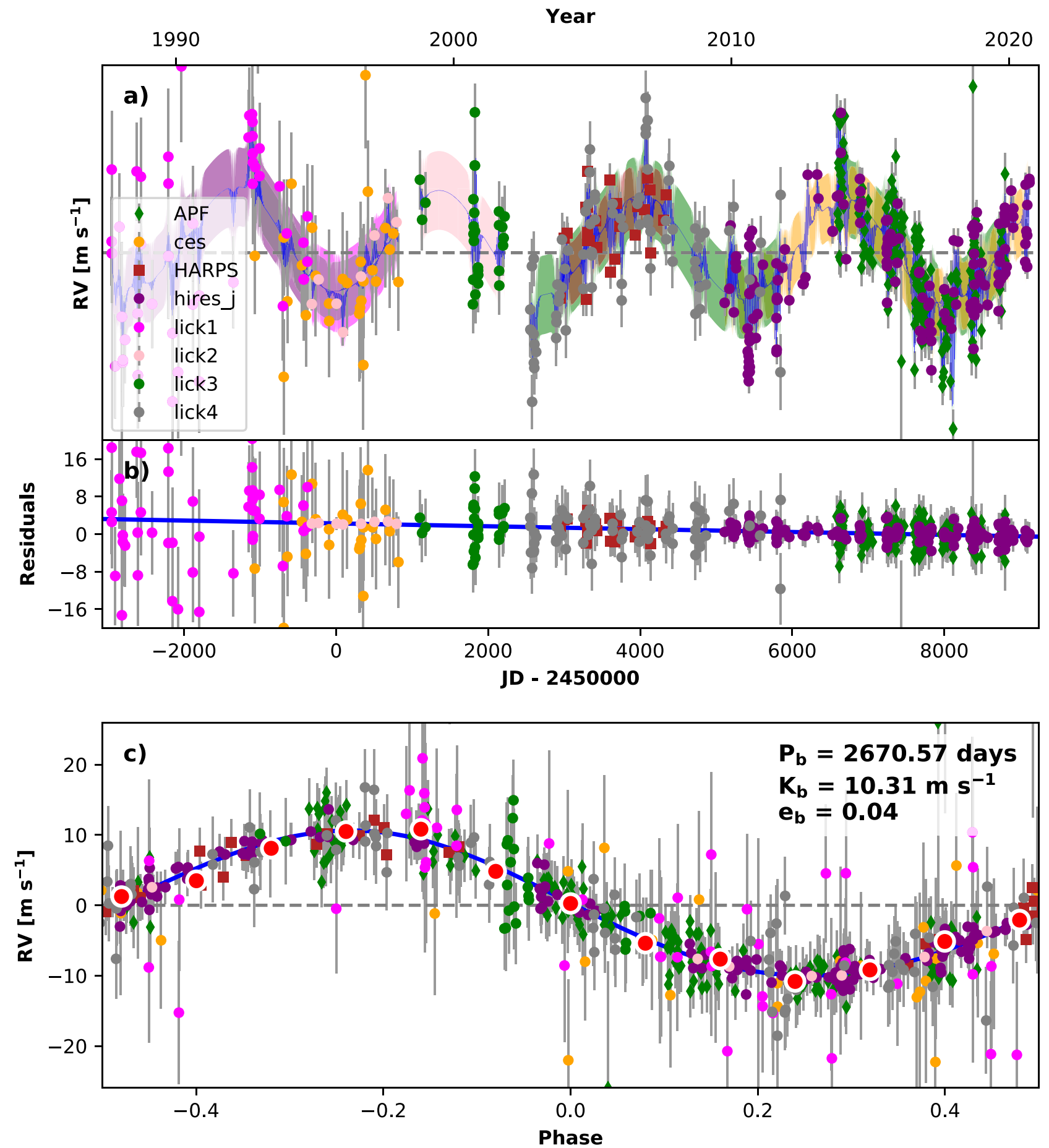

Figure 2. (a) Time series of RVs from all data sets, (b) residuals to the RV fit, and (c) phase-folded RV curve. The maximum probability one-planet model is overplotted (blue), as well as the binned data (red dots).

$x$, and $\sigma_{x}$ is the uncertainty of this estimate. Individual epochs are not combined in the main analysis of this work. The direct imaging epochs are assumed to be independent such that their log likelihoods are simply added together. While this assumption is not perfect, it should not significantly affect the upper limit as the framework marginalizes over the spatial direction which will factor in any brighter speckles. To compute $I$ from the planet mass, we use the COND evolutionary model (Baraffe et al. 2003) and we adopt an upper bound age of $800 \mathrm{Myr}$ for the system (Janson et al. 2015). To compare with this age, we also run model fits with an age of $400 \mathrm{Myr}$, corresponding to the lower bound. $\tilde{I}_{x}$ is the flux measured in the image where the planet is predicted to be based on a given set of orbital parameters.

\subsection{MCMC Results}

The fit to the astrometry and direct imaging data using the RadVel posteriors as priors is performed using the orbitize! ${ }^{20}$ package (Blunt et al. 2020). We implement a MCMC

${ }^{20}$ https://github.com/sblunt/orbitize 
Table 4

MCMC Posteriors for Different Sets of Data and Assumptions for the Age of the System

\begin{tabular}{|c|c|c|c|c|c|}
\hline & \multirow{2}{*}{$\begin{array}{l}\text { RV and Direct Imaging } \\
\qquad 800 \mathrm{Myr}\end{array}$} & \multicolumn{2}{|c|}{$\begin{array}{l}\text { RV, Astrometry, } \\
\text { and Direct Imaging }\end{array}$} & \multirow{2}{*}{$\begin{array}{l}\text { RV and Astrometry } \\
\text { No Age Assumption }\end{array}$} & \multirow{2}{*}{$\begin{array}{c}\text { RV, Astrometry (eDR3 acc.), } \\
\text { and Direct Imaging } \\
800 \mathrm{Myr}\end{array}$} \\
\hline & & $400 \mathrm{Myr}$ & $800 \mathrm{Myr}$ & & \\
\hline$\overline{M_{b}\left(M_{\mathrm{Jup}}\right)}$ & $0.74_{-0.15}^{+0.37}$ & $0.66_{-0.10}^{+0.11}$ & $0.66_{-0.09}^{+0.12}$ & $0.65_{-0.09}^{+0.10}$ & $0.64_{-0.09}^{+0.09}$ \\
\hline$a_{b}(\mathrm{au})$ & $3.53_{-0.04}^{+0.04}$ & $3.53_{-0.04}^{+0.03}$ & $3.53_{-0.04}^{+0.03}$ & $3.53_{-0.04}^{+0.04}$ & $3.52_{-0.04}^{+0.04}$ \\
\hline$e$ & $0.07_{-0.05}^{+0.07}$ & $0.07_{-0.05}^{+0.07}$ & $0.07_{-0.05}^{+0.07}$ & $0.07_{-0.05}^{+0.07}$ & $0.07_{-0.05}^{+0.07}$ \\
\hline$\omega\left(^{\circ}\right)$ & $-29.01_{-111.95}^{+107.96}$ & $-28.37_{-99.58}^{+75.90}$ & $-19.15_{-94.80}^{+88.27}$ & $-19.54_{-87.06}^{+97.09}$ & $-29.84_{-115.85}^{+104.59}$ \\
\hline$\Omega\left({ }^{\circ}\right)$ & $181.27_{-125.02}^{+124.60}$ & $195.06_{-72.21}^{+127.20}$ & $198.18_{-63.14}^{+127.29}$ & $195.08_{-73.72}^{+122.64}$ & $190.06_{-151.74}^{+108.72}$ \\
\hline$i\left(^{\circ}\right)$ & $89.15_{-43.16}^{+45.03}$ & $75.77_{-19.94}^{+29.47}$ & $78.81_{-22.41}^{+29.34}$ & $80.95_{-21.39}^{+27.55}$ & $89.70_{-25.08}^{+25.49}$ \\
\hline$\tau_{\text {peri }}$ & $0.52_{-0.33}^{+0.33}$ & $0.42_{-0.29}^{+0.34}$ & $0.35_{-0.24}^{+0.33}$ & $0.37_{-0.26}^{+0.32}$ & $0.52_{-0.36}^{+0.34}$ \\
\hline$\Delta \alpha_{H}(\mathrm{mas})$ & & $-0.33_{-0.41}^{+0.54}$ & $-0.25_{-0.47}^{+0.53}$ & $-0.21_{-0.48}^{+0.52}$ & \\
\hline$\Delta \delta_{H}(\mathrm{mas})$ & & $0.27_{-0.46}^{+0.54}$ & $0.30_{-0.47}^{+0.32}$ & $0.27_{-0.48}^{+0.32}$ & \\
\hline$P M_{H}^{\alpha}$ & & $-975.20_{-0.02}^{+0.02}$ & $-975.20_{-0.02}^{+0.02}$ & $-975.20_{-0.02}^{+0.02}$ & \\
\hline$P M_{H}^{\delta}$ & & $19.95_{-0.02}^{+0.02}$ & $19.94_{-0.02}^{+0.02}$ & $19.95_{-0.02}^{+0.02}$ & \\
\hline
\end{tabular}

(Foreman-Mackey et al. 2013) analysis to fit for the six orbital parameters and the mass of planet b. MCMC has been extensively used to do orbit fitting, e.g., first by Hou et al. (2012) and more recently by Blunt et al. (2019), Nowak et al. (2020), Hinkley et al. (2021), and Wang et al. (2021). As discussed in Section 3.2 we also fit for the mass of $\epsilon$ Eridani, as well as for the astrometric parameters $\alpha$ and $\delta$, and $\mu_{\alpha}$ and $\mu_{\delta}$. Therefore we fit for thirteen parameters: six orbital parameters, the parallax, the photocenter position vector and the proper motion vector, and the masses of the star and planet.

The knowledge of $\epsilon$ Eridani's mass is accounted with a Gaussian prior $0.82 \pm 0.02 M_{\odot}$ (Baines \& Armstrong 2011). The priors for the astrometric parameters are set to uniform distributions centered at the Hipparcos values with broad ranges of $\pm 20 \sigma$ to allow for deviations of the data from the nominal PM and photocenter position. The RV fits do not provide any constraints on the longitude of the ascending node, $\Omega$, for which we set a uniform prior distribution. As discussed in Section 3.2, although the RV fits do not constrain the inclination, $i$, we assume a sine distribution to draw correlated values for the mass, $M_{b}$, and the inclination. The fit is performed with 550 walkers, and 18,000 steps per walker.

The MCMC fits converge to yield the orbital parameters and mass posteriors shown in Table 4. Two fits are presented for both ends of the current bounds on the system's age, i.e., 400 and 800 Myr. A corner plot showing some of these and their correlations, for the $800 \mathrm{Myr}$ fit, is shown in Figure 3. A complete corner plot is shown in Appendix E.

In order to assess the constraining power of the direct imaging data, given that our data consists of nondetections, we performed an MCMC run to only fit for the astrometry data. Figure 4 show the posterior distribution compared to the prior distribution, i.e., the posteriors from the RV fit.

The use of the astrometry data results in a significantly improved constraining of the inclination of the planet with respect to the results presented in Mawet et al. (2019). Starting with a sine distribution as the prior, which is centered at $90^{\circ}$, the walkers converge onto an inclination of $i=78.81^{+29.34}$. The addition of the astrometry data also yields a different model for the mass of the companion; by using the RV and direct imaging data we obtain a distribution of $M_{b}=0.73_{-0.13}^{+0.34}$ $\mathrm{M}_{\text {Jup }}(800 \mathrm{Myr})$, by adding the astrometry, $M_{b}=0.66_{-0.09}^{+0.12}$ $\mathrm{M}_{\text {Jup }}$ (800 Myr). The astrometry data from Hipparcos and Gaia seem to favor a lower mass planet and more edge-on inclinations. We compute the most probable perturbation SMA: $\alpha_{A}=0.89$ mas, which is a factor of $\sim 2$ away from the result reported by Benedict et al. (2006), i.e., 1.88 mas. More details on the perturbed orbit can be found in Appendix D.

We add the fit to the Hipparcos-Gaia eDR3 acceleration in the last column of Table 4. The results of this fit are largely consistent with the Hipparcos IAD-Gaia DR2 fits. Two main differences can be appreciated. (1) The inclination median is $\sim 90^{\circ}$; however, the posterior probabilities converge to a similar inclination with respect to our other fits, i.e., $\sim 75^{\circ}$, and to its supplementary angle, i.e., $\sim 105^{\circ}$. This is probably because the rotation information available from the IAD is not accessible when using the PM anomalies. (2) The upper mass bound (see Figure 4) is slightly lower, which brings the mass to a lowermass solution.

The argument of periapsis, $\omega$, reported in Mawet et al. (2019), is the stellar $\omega$, which is the reason it is $\sim 180^{\circ}$ off with respect to the result presented here.

\section{Discussion}

\subsection{Debris Disk}

The interaction between the debris disk and planet in the $\epsilon$ Eridani system was thoroughly discussed in Mawet et al. (2019). Here we build on this analysis with our updated orbital constraints. $\epsilon$ Eridani's debris disk is currently known to be composed of three rings (Backman et al. 2008): a main ring from 35-90 au, an inner belt at $\sim 3 \mathrm{au}$, and an intermediate belt at $\sim 20$ au. Planet $b$ sits between the two inner belts. Extensive characterization of the disk has been carried out over the years; see Backman et al. (2008), MacGregor et al. (2015), and Booth et al. (2017). In particular, Booth et al. (2017) analysis concluded the presence of a gap in emission in the circumstellar disk between $\sim 20$ and $\sim 60$ au, which would indicate the presence of one or more companions in this range of separations. The inclination of the main ring was constrained to $34^{\circ} \pm 2$ (Booth et al. 2017).

The orbital parameters and mass of planet $b$ can set constraints on the edges of the inner belts (Wisdom 1980). We follow the same analysis as Mawet et al. (2019) given that the eccentricity of the planet is expected to be well below 0.3 , in which case the chaotic zone structure carved by the planet is 


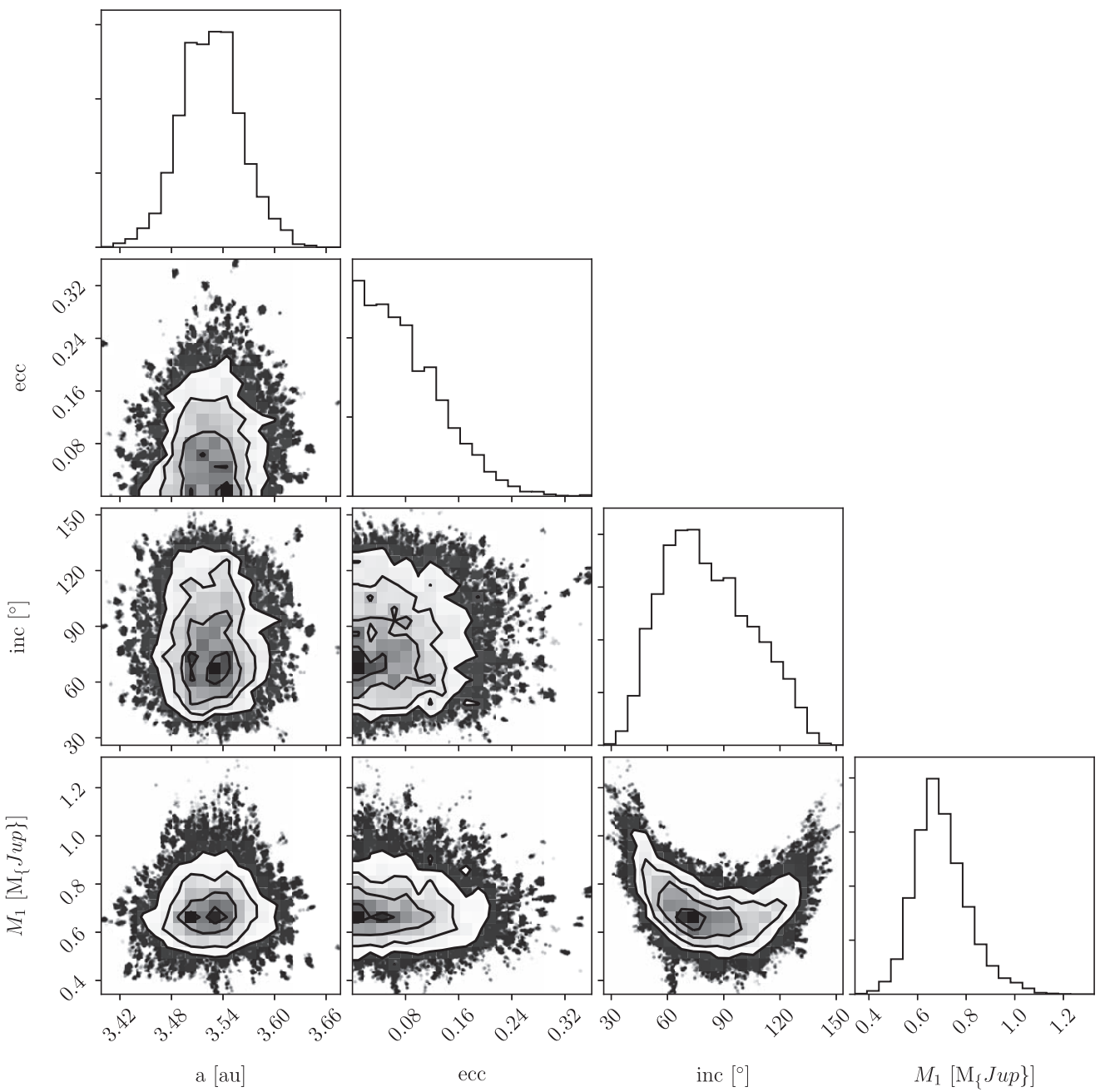

Figure 3. Corner plot of the posterior distributions and their correlation. These are the posteriors for the model fit to the RV, astrometry, and direct imaging data assuming an age of 800 Myr. We make use of corner.py (Foreman-Mackey 2016) to produce corner plots.
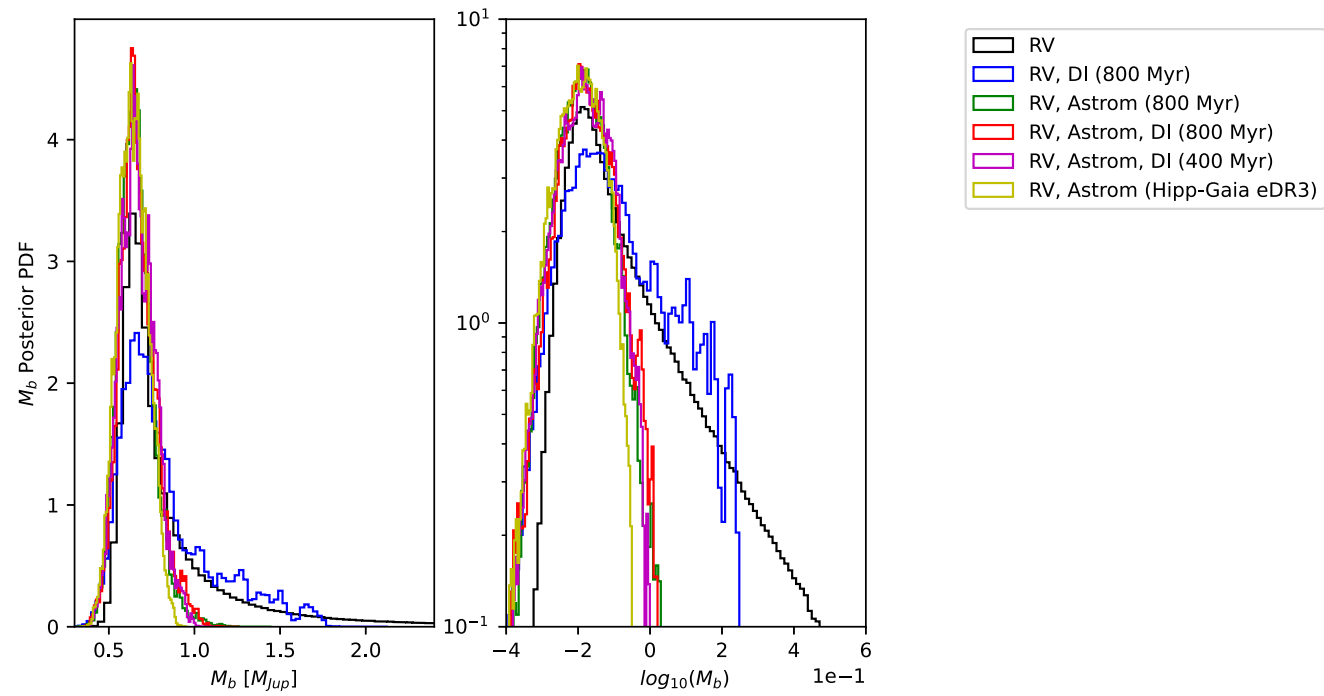

Figure 4. Mass posteriors PDF for different fits in linear (left) and logarithmic (right) scale. The astrometry data has a bigger constraining power on both ends of the mass bounds with respect to the direct imaging data available. In all fits except the RV-only fit (black) we have utilized the KDE to include the RV posteriors as priors to the fit; an artifact thus appears at the extreme of lower bound in which the distribution slightly separates from the prior. A detailed explanation of this can be found in Appendix C. However, closer to the median, the effect of adding the astrometry, for which a lower mass is allowed, is real, as it can be appreciated in the difference between the distributions with and without astrometry. 
independent of the eccentricity (Quillen \& Faber 2006). With the results of our MCMC fit (see Table 4), in particular the SMA and relative mass, we expect there to be no particles from 2.97-4.29 au. The edges are slightly moved outwards with respect to Mawet et al. (2019) since the SMA posterior of the planet is now larger, it was then reported no particles from 2.7-4.3 au. The mass posterior is lower, which reduces the width of the chaotic zone by $\sim 4 \%$.

It was concluded in Mawet et al. (2019) that the inner belt and outer ring were most likely to be self-stirred, i.e., collisions between disk particles are driven by particle-to-particle gravitational interactions, as opposed to being stirred by the presence of the planet (see Section 5.3.2. and Figure 15 in Mawet et al. 2019). The timescale for the planet to stir and shape the main ring is $\sim 1 \mathrm{Gyr}$, which, combined with the distance between the two, makes their dynamical coupling probably not significant. As for the intermediate belt, the timescales of self stirring and planet stirring were computed to be similar in the range of separations of the belt. The new orbital parameters from our MCMC fit (see Table 4), namely a lower mass for the planet and a slightly higher SMA, contribute to larger planet-stirring timescales: an $18 \%$ increase in the planet-stirring timescale estimation with respect to Mawet et al. (2019). However, this does not rule out the contribution of planet induced stirring process for the intermediate belt.

The inclination of $\epsilon$ Eridani $\mathrm{b}$ is constrained to $78.81_{-2.43}^{+29.34}$ thanks to the inclusion of the astrometry data (see Section 3.5 ). This result indicates that the planet orbit is likely inclined with respect to the main ring, for which $i=34^{\circ} \pm 2^{\circ}$, which is $\sim 2 \sigma$ away from the most probable inclination. The origin of such a mutual inclination is unknown but could possibly be due to the dynamical effects of a third body.

A mutual inclination could be causing a warping on the main ring. Indeed, the vertical warp in the $\beta$ Pictoris inner disk is believed to have been produced by its mutual inclination with $\beta$ Pictoris b (e.g., Dawson et al. 2011). Using a similar analysis as presented in Dawson et al. (2011) based on secular interactions, we find that in the case of $\epsilon$ Eridani the minimum mass of planet at 3 au to excite the inclination of dust particles in the ring at $70 \mathrm{au}$ after $800 \mathrm{Myr}$ is of $\sim 0.5 M_{\text {Jup. }}$. This indicates that planet $b$ could be in the regime of starting to drive a warp in the main ring if it is indeed misaligned with the disk plane. A coplanar solution is still allowed by the data since an inclination of $32^{\circ}$ is $\sim 1 \sigma$ away from the most probable inclination of the planet. It is worth noting that Benedict et al. (2006) yielded a solution for the inclination of the companion of $30^{\circ} .1 \pm 3.8$.

\subsection{Gaia's Future Sensitivity}

Rosa et al. (2019b) recently published the prospects for constraining mass of 51 Eridani $b$ with Gaia's final data release. They simulated sets of Gaia data with different astrometric error estimates, and found that the detection was possible only with optimistic mass and astrometric uncertainties. We performed a similar analysis computing the astrometric signal of $\epsilon$ Eridani $\mathrm{b}$ and comparing it to the sensitivity results of Figure 11 on Rosa et al. (2019b). We expect $\epsilon$ Eridani to have a similar astrometric error due to its brightness since the brightness of the star sets the uncertainty in the scans. We get an estimate for this at Lindegren et al. (2018): 50 $\mu$ as.

We find that the amplitude of the astrometric signal for $\epsilon$ Eridani $b$ is an order of magnitude stronger than that of 51
Eridani $\mathrm{b}$. Indeed, the shorter distance to the system and the period the planet both favorable factors for a stronger astrometric signal. For the nominal Gaia mission span of $5 \mathrm{yr}$ and for an astrometric uncertainty in the scans of $50 \mu a, \epsilon$ Eridani $\mathrm{b}$ is detectable at $\sim 1 M_{\text {Jup }}$, which falls on the higher end of our mass posterior probability. However, for the extended mission span of $8 \mathrm{yr}$, for which 51 Eridani is only detectable for a high mass estimate, $\epsilon$ Eridani $\mathrm{b}$ is readily detectable even at $\sim 0.5 M_{\text {Jup }}$, which is on the lower than the median mass of the posterior probability presented in this paper.

The final data release of Gaia's mission is a particularly exciting prospect for the exoplanet science field. Gaia final release will probably have access to constraining the dynamic mass of $\epsilon$ Eridani $\mathrm{b}$. However, as the work presented in this paper aims at showing, it is by using this data combined with other observations that the best science is attainable.

\subsection{Advantages of Combining Different Methods}

The results presented in this paper are another example of the power of combining different methods to constrain a system's characteristics. By adding the astrometry data, we have identified new constraints for the inclination and longitude of the ascending node, both of which are inaccessible to an RV-orbit fit. The direct imaging data, although it being a nondetection, sets upper limits on the mass. The distribution of most likely planet positions shown in Figure 5 and how it prefers certain areas and fluxes is no coincidence; the MCMC walkers converge easier where the estimated flux from the coronagraph images is higher.

Although the constraints on the position shown in Figure 5 are far from ideal, direct imaging planet hunters will take advantage of any position knowledge however small it may be. Indeed, data reduction techniques in direct imaging greatly benefit from a prior knowledge of the position of the object. For instance, in PCA(Soummer et al. 2012) based methods, a great deal of speckle subtraction power is gained by treating the data by patches; knowing where the planet is more likely to be reduces the computing time and allows the algorithm to focus on a constrained area of interest.

The synergies between RV, astrometry, and direct imaging data are currently being explored, and more work is being done in this direction (GRAVITY Collaboration et al. 2020).

\subsection{Prospects for a Direct Detection with JWST}

The James Webb Space Telescope (JWST) will provide the community with unprecedented capabilities to do infrared exoplanet science. In this section we discuss the prospect for a detection of $\epsilon$ Eridani b with JWST's NIRSPEC and MIRI instruments. In Figure 6 we show the probability contour for the position of the planet at an epoch in JWST's Cycle 1.

\subsubsection{MIRI}

The midinfrared instrument (MIRI) aboard JWST is equipped with a coronagraph, and is expected to reach $10^{-4}$ levels of raw contrast at $\lambda=10 \mu \mathrm{m}$ (mode F1065C) with conventional star subtraction (Boccaletti et al. 2015). We performed a more sophisticated data reduction as was done for MIRI in Beichman et al. (2020) to get a more accurate representation of the expected performance of MIRI on $\epsilon$ Eridani. We use the IDL library MIRImS IM, ${ }^{21}$ and the wave

\footnotetext{
${ }^{21}$ https://jwst.fr/wp/?p=30
} 

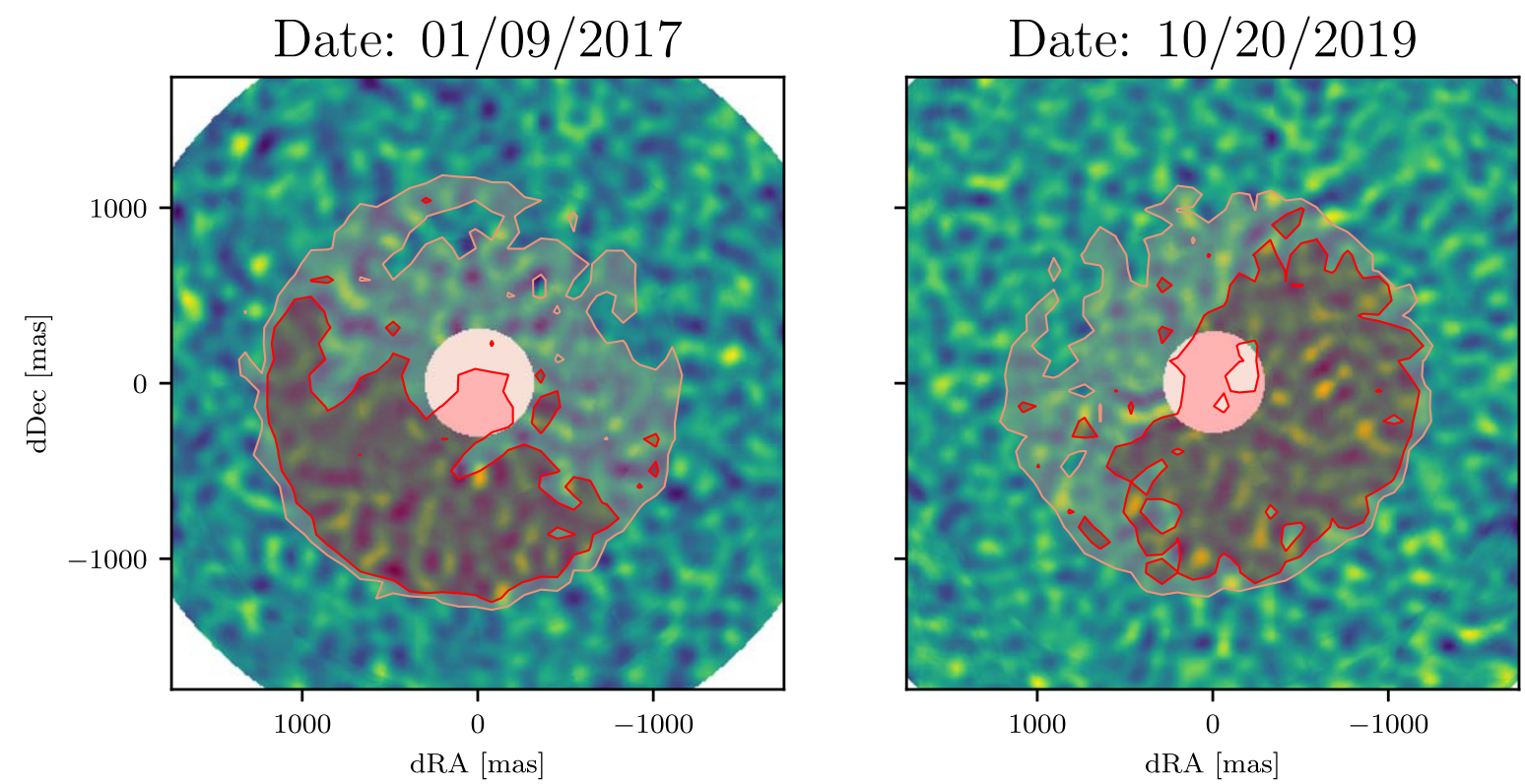

Figure 5. Keck/NIRC2 reduced data for two of the nine observing epochs (see Table 2), the 1 and $2 \sigma$ contours of the posteriors of the position are overplotted. The posteriors are for the model fit to the RV, astrometry and direct imaging data assuming an age of $800 \mathrm{Myr}$. The addition of the astrometry and direct imaging data breaks the degeneracy on the inclination, and the position of the companion is better constrained.

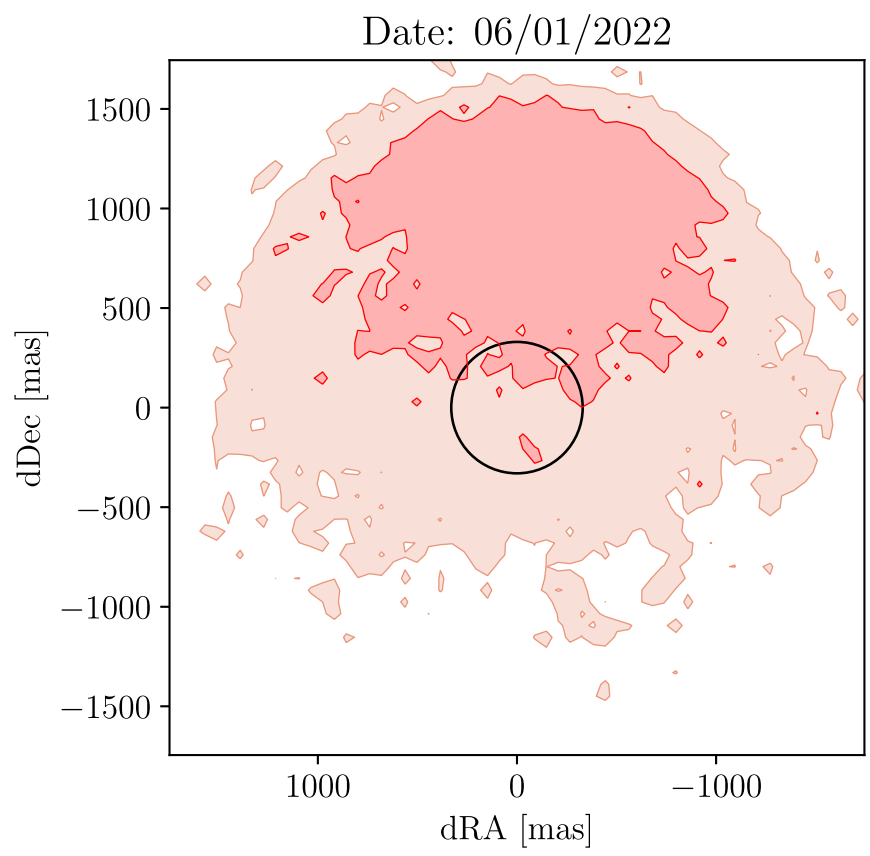

Figure 6. 1 and $2 \sigma$ contours of the planet's position at a possible JWST epoch. This information can be useful for observers; for instance, when using the $4 \mathrm{QPM}$ coronagraph, the user will want to avoid the gaps falling on the most probable position of planet $b$. The black circle indicates the inner working angle of MIRI's F1065 mode Boccaletti et al. (2015). The posteriors used for this contours are taken from the fit to the RV, astrometry, and direct imaging data, assuming an $800 \mathrm{Myr}$ age.

front error drift predictions presented in Perrin et al. (2018). We make use of as much diversity as possible from the jitter of the telescope to perform a reference star subtraction based on PCA (Soummer et al. 2012). A more detailed description of the data processing can be found in Beichman et al. (2020).

In Figure 7 we show some simulation results for MIRI observations. We find that, even for a perfect pointing accuracy of the telescope, MIRI would require $\sim 75 \mathrm{hr}$ to reach $\mathrm{SNR}>5$.

\subsubsection{NIRSpec}

A new avenue to imaging $\epsilon$ Eridani $b$ is by using the moderate spectral resolution integral field spectroscopy mode of NIRSPEC (G395H/F290LP, with its $3^{\prime \prime} \times 3^{\prime \prime}$ field of view). Atmospheric models predict a large excess emission around $4.5 \mu \mathrm{m}$ from the atmosphere of exoplanets such as $\epsilon$ Eridani $\mathrm{b}$ (Marley et al. 2018). Ground-based medium resolution spectrographs $(R \sim 4000)$ like OSIRIS and SINFONI have made clear detections of exoplanets as close as 0 ". 4 from their star detector water and carbon monoxyde (Konopacky et al. 2013; Barman et al. 2015; Hoeijmakers et al. 2018; Wilcomb et al. 2020). The advantage of a higher spectral resolution is the possibility to subtract the starlight continuum with a high-pass filter and then use cross-correlation techniques to detect the molecular spectral signature of the planet. Like NIRSpec, these instruments were not designed for exoplanet detection, but the increased resolution can overcome a lack of a coronagraph and achieve comparable, if not better, detections of imaged exoplanets. Furthermore, the fact that these are spectroscopic detections opens up rich capabilities in atmospheric characterization that are simply not possible through imaging alone. Houllé et al. (2021) demonstrated the power of high spectral resolution integral spectroscopy in the context of HARMONI, a first light instrument to the extremely large telescope, showing that it could detect planets 10 times fainter than angular differential imaging. NIRSpec is expected to excel at this technique thanks to the stability of a space observatory and the absence of variable telluric lines, which can be the source of spurious detection of molecules as discussed in Petit dit de la Roche et al. (2018).

To assess the feasibility of detecting these planets, we simulated NIRSpec observations with the JWST exposure time calculator (ETC) and implemented a forward modeling approach similar to Hoeijmakers et al. (2018) and Ruffio et al. (2019) to NIRSpec in which a starlight and a planet model are jointly fitted. The planet model consists of a Sonora atmospheric (Marley et al. 2018) modulated by the transmission of 

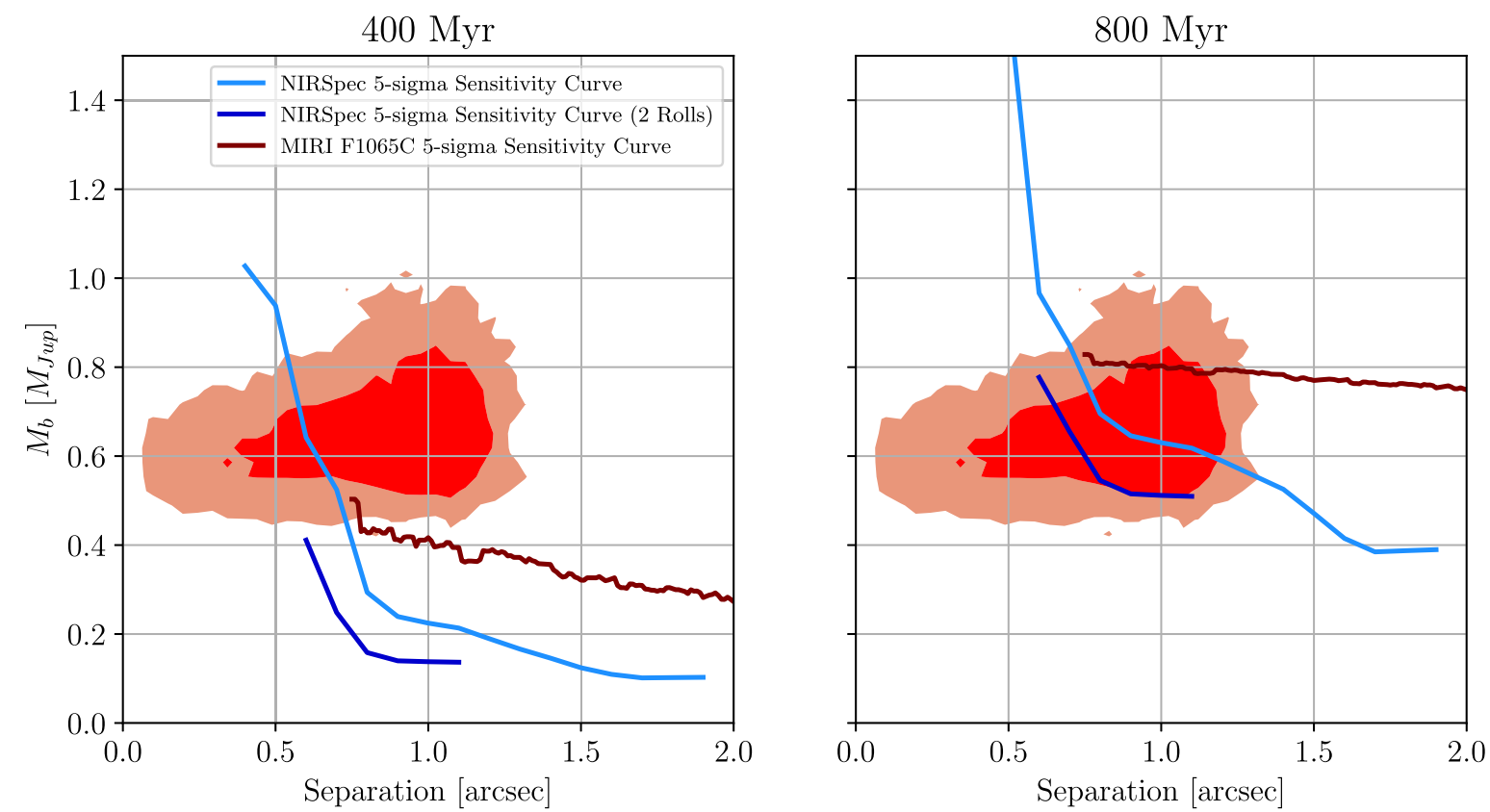

Figure 7. Expected $5 \sigma$ sensitivity for NIRSpec and MIRI using our data reduction techniques, compared to the expected location and mass of the companion; red: 1 and $2 \sigma$ contours at an epoch close to the expected maximum elongation, i.e., January 2024. The upper sensitivity curve for NIRSpec corresponds to 2 hr of exposure times, while the two-roll case corresponds to a total of $4 \mathrm{hr}$ with a $30^{\circ}$ pupil rotation between two rolls to mitigate the effect of the diffraction spikes of the JWST PSF. The MIRI simulations require $\sim 75 \mathrm{hr}$ of exposure time to get enough signal to noise. These results indicate that NIRSpec is the most sensitive instrument for this science case.

the instrument. The same simulation is used to derive the sensitivity of NIRSpec as a function of separation, shown in Figure 7. The JWST ETC does not include many of the likely source of errors that will affect the calibration of the data so the final sensitivity remains uncertain. However, cross-correlation techniques are not sensitive to speckle variability and chromaticity, or telescope pointing precision unlike conventional speckle subtraction techniques. The observations are dominated by the photon noise from the diffracted starlight, so it is critical to minimize the effect of the diffraction spikes in the JWST PSF. They are more than an order of magnitude brighter than the rest of the PSF at a given separation. To avoid chance alignments of the planets with the diffraction spikes, two visits per star with a $30^{\circ}$ pupil rotation can be used to double the average sensitivity of the observation, which is twice as efficient as simply increasing the integration time (Figure 7). Even in the fastest reading mode and shortest available integration time, the core of the PSF will heavily saturate around $0 . \prime 6$, which limits the inner working angle. We note that the planets only emit toward the redder part of the band $(4.2 \mu \mathrm{m})$ where the starlight is dimmer, so any wavelength shorter than $4.2 \mu \mathrm{m}$ is allowed to saturate with no consequence. Any detector persistence in pixels previously saturated in an earlier dither position will appear like slightly elevated stellar signal, and will naturally be removed by the high-pass filtering as if it were speckle noise.

\subsection{Prospective for a Detection in Reflected Light with the Roman Coronagraph Instrument}

Carrión-González et al. (2021) assessed the potential of detecting reflected light from a set of exoplanets in nearby systems with the Roman coronagraph instrument (CGI). In particular, $\epsilon$ Eridani stands out as a particularly notable, yet risky target; like most targets for the Roman CGI, it would require thousands of hours to get a detection. They conclude that $\epsilon$ Eridani b would be Roman-accessible at a probability of $57.99 \%$, in the optimistic case, and $51.29 \%$ in the pessimistic case. However, they argue that the inclination "is the key factor affecting the detectability of this planet." Indeed, as it can be seen in their Figure C.5, a more edge-on orbit is more favorable to avoiding the outer working angle. Our result for the inclination, $i=78.81_{-23.41}^{+29.34}$, indicates that the orbit should be more favorable for detection, since Carrión-González et al. (2021) assume face on as the most probable orbit.

\subsection{Prospects for Ground-based Observatories}

A recent publication by Pathak et al. (2021) presented new observations of $\epsilon$ Eridani at $10 \mu \mathrm{m}$ with the VLT/VISIR. They obtained comparable sensitivities to the Keck/NIRC2 results presented here. They claim that a sensitivity to $1 M_{\text {Jup }}$ can be attained with $70 \mathrm{hr}$ of exposure time, assuming an age of $700 \mathrm{Myr}$ and the current setup for the instrument. Unfortunately, the results presented here indicate that the mass is likely lower than $1 M_{\text {Jup }}$. However, there are envisioned ways to upgrading VISIR which would improve its sensitivity at smaller separations (Kasper et al. 2019).

Although a formidable task for current ground-based observatories, imaging $\epsilon$ Eridani b should be much easier with future 30-meter class telescopes. High-contrast imaging at L, $\mathrm{M}$, and $\mathrm{N}$ bands with METIS at the ELT will essentially be background limited at the angular separation of $\epsilon$ Eridani $b$ (Carlomagno et al. 2020). METIS is expected to have access to Earth-like planets around $\alpha$ Centauri A with $5 \mathrm{hr}$ of exposure time $\mathrm{N}$ band (Brandl et al. 2021), which would make $\epsilon$ Eridani $\mathrm{b}$ detectable in the order of minutes. Similarly, the TMT with its second generation instrument PSI is expected to reach $10^{-8}$ final contrast at $2 \lambda / D$ (Fitzgerald et al. 2019), well above the sensitivity needed to image $\epsilon$ Eridani b. 


\section{Conclusion}

We combine observations of $\epsilon$ Eridani from three different methods: RVs spanning three decades, the combined astrometry data from Hipparcos IAD and GRD2, and vortex coronagraph images with Keck/NIRC2. We perform a fit to this data using MCMC and obtain the best constraints to date for the orbital parameters and mass of $\epsilon$ Eridani b. Namely, a lower-mass posterior with respect to previous analysis (Mawet et al. 2019), $M_{b}=0.66_{-0.09}^{+0.12} \mathrm{M}_{\mathrm{Jup}}$, and new constraints for the inclination, $i=78.81_{-22.41}^{+29.34}$. The new inclination seems to indicate that the planet orbit is not coplanar with the main ring structure, at $i=34^{\circ} \pm 2^{\circ}$. Our results are consistent with a small eccentricity, and we improved the accuracy of the time of conjunction to $\sim 81$ days.

These improved constraints translate in a more confident prediction of the position at any epoch as (see Figures 5 and 6). We show how this information can be useful when planning the observing strategy, and data reduction, with future missions like the JWST. The JWST is a particularly exciting prospect: we show how NIRSpec could obtain a detection with a reasonable exposure time of just a few hours. More work is expected to be done in this regard. Another exciting landmark for the field of exoplanetary science is the final release of Gaia's data; we show how, with the expected sensitivity, Gaia will most likely have access to a dynamic-mass measurement of $\epsilon$ Eridani b.

In this paper, we show that the combination of data sets from different observing methods has the power to yield previously inaccessible planetary characteristics from the elusive $\epsilon$ Eridani b. As more RV data continues to be collected, and RV facilities and instruments continue to be improved, plus the prospects of Gaia's final data release, and future coronagraph images, the prospects for studying this planet are promising.

This work was partially supported by the National Science Foundation AST-ATI Grant 1710210. The material is based upon work supported by NASA under award No. 80NSSC20K0624. J.J.W. is supported by the Heising-Simons Foundation 51 Pegasi b Fellowship. J.J.W. thanks Rob de Rosa and Eric Nielsen for helpful discussions. P.D. is supported by a National Science Foundation (NSF) Astronomy and Astrophysics Postdoctoral Fellowship under award AST-1903811. M.R. is supported by the National Science Foundation Graduate Research Fellowship Program under grant No. DGE-1752134 Part of this work has received funding from the European Research Council (ERC) under the European Union's Horizon 2020 research and innovation program (grant agreement No. 819155), and by the Wallonia-Brussels Federation (grant for Concerted Research Actions). We thank to Christopher Mendillo for useful discussions.

Software: pyKLIP (Wang et al. 2015), Scipy(Jones et al. 2001), orbitize! (Blunt et al. 2020), emcee (ForemanMackey et al. 2013), corner.py (Foreman-Mackey 2016), RVSearch (Rosenthal et al. 2021), Astropy (Astropy Collaboration et al. 2013), Matplotlib (Hunter 2007).

\section{Appendix A Radial-velocity Measurements}

The new RV measurements are shown in Table 5.
Table 5

Radial Velocities

\begin{tabular}{|c|c|c|c|}
\hline $\begin{array}{l}\text { Time } \\
\text { (JD) }\end{array}$ & $\begin{array}{c}\mathrm{RV} \\
\left(\mathrm{m} \mathrm{s}^{-1}\right)\end{array}$ & $\begin{array}{l}\text { RV Unc. } \\
\left(\mathrm{m} \mathrm{s}^{-1}\right)\end{array}$ & Inst. \\
\hline $2,457,830.7206$ & -13.32 & 1.10 & hires $_{j}$ \\
\hline $2,457,957.0042$ & -9.42 & 2.67 & apf \\
\hline $2,457,957.0049$ & -18.75 & 2.72 & apf \\
\hline $2,457,957.0055$ & -18.07 & 2.86 & apf \\
\hline $2,457,971.9799$ & -19.64 & 2.61 & apf \\
\hline $2,457,971.9806$ & -21.74 & 2.51 & apf \\
\hline $2,457,971.9814$ & -20.58 & 2.53 & apf \\
\hline $2,457,975.9705$ & -29.90 & 2.50 & apf \\
\hline $2,457,975.9712$ & -26.60 & 2.55 & apf \\
\hline $2,457,975.9720$ & -26.59 & 2.45 & apf \\
\hline $2,457,983.0029$ & -25.13 & 2.36 & apf \\
\hline $2,457,983.0036$ & -28.48 & 2.19 & apf \\
\hline $2,457,983.0044$ & -23.69 & 2.33 & apf \\
\hline $2,457,991.9333$ & -19.97 & 2.54 & apf \\
\hline $2,457,991.9342$ & -19.61 & 2.37 & apf \\
\hline $2,457,991.9350$ & -20.31 & 2.42 & apf \\
\hline $2,457,993.0151$ & -23.62 & 2.13 & apf \\
\hline $2,457,993.0158$ & -30.11 & 2.32 & apf \\
\hline $2,457,993.0166$ & -21.83 & 2.05 & apf \\
\hline $2,458,000.1544$ & -4.85 & 0.90 & hires $_{j}$ \\
\hline $2,458,001.1525$ & -4.85 & 0.95 & hires $_{j}$ \\
\hline $2,458,003.1521$ & -9.65 & 0.92 & hires $_{j}$ \\
\hline $2,458,011.8651$ & -6.39 & 3.20 & apf \\
\hline $2,458,011.8658$ & -14.24 & 3.06 & apf \\
\hline $2,458,011.8665$ & -8.81 & 2.98 & apf \\
\hline $2,458,011.9773$ & -3.93 & 2.69 & apf \\
\hline $2,458,011.9779$ & -9.78 & 2.72 & apf \\
\hline $2,458,011.9786$ & -13.28 & 2.60 & apf \\
\hline $2,458,024.9756$ & -9.99 & 2.20 & apf \\
\hline $2,458,024.9763$ & -10.66 & 2.23 & apf \\
\hline $2,458,024.9770$ & -9.56 & 2.46 & apf \\
\hline $2,458,027.8153$ & -18.68 & 3.08 & apf \\
\hline $2,458,027.8173$ & -16.81 & 3.07 & apf \\
\hline $2,458,027.8191$ & -19.40 & 2.79 & apf \\
\hline $2,458,027.9419$ & -18.22 & 4.21 & apf \\
\hline $2,458,027.9427$ & -18.13 & 2.92 & apf \\
\hline $2,458,027.9434$ & -18.38 & 3.45 & apf \\
\hline $2,458,029.9391$ & -11.04 & 1.07 & hires $_{j}$ \\
\hline $2,458,031.9469$ & -16.01 & 2.19 & apf \\
\hline $2,458,031.9477$ & -15.41 & 2.34 & apf \\
\hline $2,458,031.9485$ & -17.23 & 2.22 & apf \\
\hline $2,458,032.9383$ & -9.09 & 2.28 & apf \\
\hline $2,458,032.9392$ & -6.62 & 2.20 & apf \\
\hline $2,458,032.9400$ & -10.73 & 2.25 & apf \\
\hline $2,458,039.8294$ & -12.02 & 2.29 & apf \\
\hline $2,458,039.8301$ & -12.76 & 2.25 & apf \\
\hline $2,458,039.8309$ & -10.73 & 2.37 & apf \\
\hline $2,458,040.9596$ & -14.86 & 2.28 & apf \\
\hline $2,458,040.9604$ & -15.63 & 2.28 & apf \\
\hline $2,458,040.9612$ & -15.16 & 2.30 & apf \\
\hline $2,458,041.9011$ & -17.38 & 2.27 & apf \\
\hline $2,458,041.9019$ & -20.79 & 2.03 & apf \\
\hline $2,458,041.9027$ & -20.04 & 2.10 & apf \\
\hline $2,458,051.7346$ & -9.28 & 3.29 & apf \\
\hline $2,458,051.7353$ & -13.60 & 3.34 & apf \\
\hline $2,458,051.7360$ & -10.66 & 3.23 & apf \\
\hline $2,458,063.8317$ & -9.47 & 3.22 & apf \\
\hline $2,458,063.8324$ & -18.04 & 3.19 & apf \\
\hline $2,458,063.8332$ & -11.10 & 3.49 & apf \\
\hline $2,458,065.9048$ & -11.21 & 1.16 & hires $_{j}$ \\
\hline $2,458,080.8503$ & -28.17 & 2.22 & apf \\
\hline $2,458,080.8511$ & -28.91 & 2.42 & apf \\
\hline $2,458,080.8519$ & -23.24 & 2.28 & apf \\
\hline
\end{tabular}


Table 5

(Continued)

\begin{tabular}{|c|c|c|}
\hline $\begin{array}{l}\text { Time } \\
\text { (JD) }\end{array}$ & $\begin{array}{c}\mathrm{RV} \\
\left(\mathrm{m} \mathrm{s}^{-1}\right)\end{array}$ & $\begin{array}{l}\text { RV Unc. } \\
\left(\mathrm{m} \mathrm{s}^{-1}\right)\end{array}$ \\
\hline $2,458,086.7355$ & -10.09 & 2.62 \\
\hline $2,458,086.7362$ & -17.91 & 2.75 \\
\hline $2,458,086.7369$ & -13.81 & 2.65 \\
\hline $2,458,088.6965$ & -8.24 & 2.75 \\
\hline $2,458,088.6971$ & -8.44 & 2.70 \\
\hline $2,458,088.6978$ & -6.73 & 2.93 \\
\hline $2,458,088.8101$ & -3.59 & 2.29 \\
\hline $2,458,088.8108$ & -7.83 & 2.24 \\
\hline $2,458,088.8116$ & -3.13 & 2.32 \\
\hline $2,458,091.9103$ & -12.71 & 1.17 \\
\hline $2,458,115.6819$ & -32.50 & 2.89 \\
\hline $2,458,115.6826$ & -37.07 & 2.71 \\
\hline $2,458,115.6832$ & -38.80 & 2.76 \\
\hline $2,458,119.7231$ & -11.88 & 3.31 \\
\hline $2,458,119.7238$ & -9.47 & 2.80 \\
\hline $2,458,119.7245$ & -8.95 & 3.07 \\
\hline $2,458,124.8560$ & 4.97 & 1.04 \\
\hline $2,458,125.6912$ & -5.43 & 0.96 \\
\hline $2,458,131.6676$ & -15.23 & 2.81 \\
\hline $2,458,131.6683$ & -3.72 & 2.92 \\
\hline $2,458,131.6690$ & -9.99 & 2.99 \\
\hline $2,458,154.7030$ & -7.90 & 1.15 \\
\hline $2,458,156.6999$ & -6.81 & 2.40 \\
\hline $2,458,156.7009$ & -6.12 & 2.39 \\
\hline $2,458,156.7018$ & -2.55 & 2.40 \\
\hline $2,458,175.6205$ & -4.60 & 2.54 \\
\hline $2,458,175.6222$ & -9.71 & 2.26 \\
\hline $2,458,175.6236$ & -5.39 & 2.21 \\
\hline $2,458,181.7462$ & 4.72 & 1.36 \\
\hline $2,458,343.0008$ & -3.22 & 2.55 \\
\hline $2,458,343.0017$ & -4.83 & 2.48 \\
\hline $2,458,343.0025$ & -5.67 & 2.54 \\
\hline $2,458,346.1447$ & -7.86 & 0.93 \\
\hline $2,458,350.1449$ & -8.76 & 1.00 \\
\hline $2,458,356.9155$ & -1.36 & 2.23 \\
\hline $2,458,356.9162$ & -4.72 & 2.17 \\
\hline $2,458,356.9170$ & 2.84 & 2.08 \\
\hline $2,458,357.9251$ & -12.01 & 2.49 \\
\hline $2,458,357.9258$ & -16.35 & 2.32 \\
\hline $2,458,357.9266$ & -17.44 & 2.35 \\
\hline $2,458,359.9026$ & -19.24 & 2.44 \\
\hline $2,458,359.9035$ & -25.73 & 2.50 \\
\hline $2,458,359.9043$ & -20.22 & 2.51 \\
\hline $2,458,367.0466$ & 7.57 & 1.00 \\
\hline $2,458,369.0367$ & -8.97 & 2.19 \\
\hline $2,458,369.0375$ & -7.22 & 2.23 \\
\hline $2,458,369.0382$ & -6.04 & 2.14 \\
\hline $2,458,370.0423$ & -18.25 & 2.27 \\
\hline $2,458,370.0430$ & -18.08 & 2.26 \\
\hline $2,458,370.0437$ & -20.25 & 2.41 \\
\hline $2,458,377.0051$ & 32.40 & 38.87 \\
\hline $2,458,379.0183$ & -4.80 & 2.39 \\
\hline $2,458,379.0189$ & -5.50 & 2.50 \\
\hline $2,458,379.0196$ & -7.38 & 2.29 \\
\hline $2,458,384.0611$ & -16.79 & 1.01 \\
\hline $2,458,386.0254$ & -9.85 & 1.06 \\
\hline $2,458,387.0174$ & 0.19 & 1.08 \\
\hline $2,458,387.9630$ & 3.39 & 2.26 \\
\hline $2,458,387.9637$ & 1.27 & 2.42 \\
\hline $2,458,387.9645$ & -0.11 & 2.23 \\
\hline $2,458,387.9940$ & 8.09 & 1.10 \\
\hline $2,458,390.0029$ & 1.72 & 2.50 \\
\hline $2,458,390.0042$ & 2.69 & 2.37 \\
\hline
\end{tabular}

Table 5

(Continued)
Inst. apf

apf

apf

apf

apf

apf

apf

apf

hires $_{j}$

apf

apf

apf

apf

apf

ires ${ }_{j}$

hires $_{j}$

apf

apf

apf

hires $_{j}$

apf

apf

apf

apf

apf

hires $_{j}$

apf

apf

apf

hires $_{j}$

ires $_{j}$

apf

apf

apf

apf

apf

apf

apf

apf

hires $_{j}$

apf

apf

apf

apf

apf

apf

apf

apf

apf

apf

hires $_{j}$

hires $_{j}$

apf

apf

apf

apf

\begin{tabular}{|c|c|c|c|}
\hline $\begin{array}{l}\text { Time } \\
\text { (JD) }\end{array}$ & $\begin{array}{c}\mathrm{RV} \\
\left(\mathrm{m} \mathrm{s}^{-1}\right)\end{array}$ & $\begin{array}{l}\text { RV Unc. } \\
\left(\mathrm{m} \mathrm{s}^{-1}\right)\end{array}$ & Inst. \\
\hline $2,458,390.0042$ & -2.89 & 2.47 & apf \\
\hline $2,458,390.0479$ & 6.12 & 0.96 & hires $_{j}$ \\
\hline $2,458,390.9784$ & -3.06 & 2.26 & apf \\
\hline $2,458,390.9792$ & 3.35 & 2.41 & apf \\
\hline $2,458,390.9801$ & 2.41 & 2.38 & apf \\
\hline $2,458,392.0595$ & 2.27 & 1.08 & hires $_{j}$ \\
\hline $2,458,394.0773$ & -7.38 & 1.04 & hires $_{j}$ \\
\hline $2,458,396.0349$ & -10.04 & 1.19 & hires $_{j}$ \\
\hline $2,458,396.9693$ & -3.90 & 1.23 & hires $_{j}$ \\
\hline $2,458,398.8491$ & 4.51 & 3.30 & apf \\
\hline $2,458,398.8499$ & -2.61 & 3.38 & apf \\
\hline $2,458,398.8506$ & -2.75 & 3.02 & apf \\
\hline $2,458,398.9204$ & 0.82 & 2.90 & apf \\
\hline $2,458,398.9211$ & 4.59 & 3.02 & apf \\
\hline $2,458,398.9217$ & -5.49 & 2.89 & apf \\
\hline $2,458,399.9857$ & -0.15 & 2.55 & apf \\
\hline $2,458,399.9866$ & 1.95 & 2.51 & apf \\
\hline $2,458,399.9874$ & 1.36 & 2.44 & apf \\
\hline $2,458,400.9738$ & 0.34 & 2.46 & apf \\
\hline $2,458,400.9746$ & -0.60 & 2.37 & apf \\
\hline $2,458,400.9753$ & 0.62 & 2.41 & apf \\
\hline $2,458,410.9247$ & -12.64 & 2.46 & apf \\
\hline $2,458,410.9254$ & -7.35 & 2.31 & apf \\
\hline $2,458,410.9261$ & -8.92 & 2.35 & apf \\
\hline $2,458,414.8894$ & 9.86 & 2.17 & apf \\
\hline $2,458,414.8902$ & 4.71 & 2.10 & apf \\
\hline $2,458,414.8909$ & 1.61 & 2.29 & apf \\
\hline $2,458,418.8942$ & -21.68 & 2.42 & apf \\
\hline $2,458,418.8950$ & -25.37 & 2.40 & apf \\
\hline $2,458,418.8958$ & -20.21 & 2.35 & apf \\
\hline $2,458,419.9035$ & -13.62 & 2.99 & apf \\
\hline $2,458,419.9045$ & -11.57 & 2.79 & apf \\
\hline $2,458,419.9067$ & -11.59 & 2.07 & apf \\
\hline $2,458,426.9223$ & 10.59 & 1.04 & hires $_{j}$ \\
\hline $2,458,439.0088$ & 7.54 & 1.28 & hires $_{j}$ \\
\hline $2,458,443.8821$ & -10.76 & 1.18 & hires $_{j}$ \\
\hline $2,458,443.8827$ & -6.02 & 1.02 & hires $_{j}$ \\
\hline $2,458,443.8832$ & -10.14 & 1.22 & hires $_{j}$ \\
\hline $2,458,462.8538$ & -2.77 & 1.02 & hires $_{j}$ \\
\hline $2,458,462.8544$ & -1.14 & 1.04 & hires $_{j}$ \\
\hline $2,458,462.8549$ & -2.01 & 1.01 & hires $_{j}$ \\
\hline $2,458,476.7717$ & -3.32 & 1.02 & hires $_{j}$ \\
\hline $2,458,476.7722$ & -2.91 & 1.05 & hires $_{j}$ \\
\hline $2,458,476.7727$ & -3.41 & 0.97 & hires $_{j}$ \\
\hline $2,458,480.7128$ & -4.31 & 2.48 & apf \\
\hline $2,458,480.7139$ & 2.18 & 2.60 & apf \\
\hline $2,458,480.7150$ & -0.89 & 2.54 & apf \\
\hline $2,458,487.6420$ & -4.39 & 2.37 & apf \\
\hline $2,458,487.6427$ & -4.97 & 2.28 & apf \\
\hline $2,458,487.6434$ & -6.74 & 2.41 & apf \\
\hline $2,458,488.6304$ & -16.61 & 2.84 & apf \\
\hline $2,458,488.6311$ & -17.42 & 2.53 & apf \\
\hline $2,458,488.6317$ & -15.66 & 2.40 & apf \\
\hline $2,458,490.7571$ & 2.75 & 1.04 & hires $_{j}$ \\
\hline $2,458,490.7577$ & 0.01 & 1.05 & hires $_{j}$ \\
\hline $2,458,490.7582$ & -1.50 & 1.06 & hires $_{j}$ \\
\hline $2,458,532.7200$ & -4.04 & 1.12 & hires $_{j}$ \\
\hline $2,458,568.7106$ & -7.45 & 1.08 & hires $_{j}$ \\
\hline $2,458,568.7112$ & -8.92 & 1.17 & hires $_{j}$ \\
\hline $2,458,568.7117$ & -7.60 & 1.12 & hires $_{j}$ \\
\hline $2,458,569.7117$ & -7.98 & 1.00 & hires $_{j}$ \\
\hline $2,458,569.7123$ & -7.88 & 1.10 & hires $_{j}$ \\
\hline $2,458,569.7128$ & -8.14 & 1.02 & hires $_{j}$ \\
\hline
\end{tabular}


Table 5

(Continued)

\begin{tabular}{|c|c|c|c|}
\hline $\begin{array}{l}\text { Time } \\
\text { (JD) }\end{array}$ & $\begin{array}{c}\mathrm{RV} \\
\left(\mathrm{m} \mathrm{s}^{-1}\right)\end{array}$ & $\begin{array}{l}\text { RV Unc. } \\
\left(\mathrm{m} \mathrm{s}^{-1}\right)\end{array}$ & Inst. \\
\hline $2,458,714.1492$ & -9.22 & 1.08 & hires $_{j}$ \\
\hline $2,458,715.1508$ & -1.96 & 0.93 & hires $_{j}$ \\
\hline $2,458,716.1499$ & -3.73 & 0.89 & hires $_{j}$ \\
\hline $2,458,723.1549$ & 8.30 & 0.96 & hires $_{j}$ \\
\hline $2,458,724.1550$ & 1.85 & 0.93 & hires $_{j}$ \\
\hline $2,458,732.0204$ & -7.99 & 2.41 & apf \\
\hline $2,458,732.0230$ & -9.53 & 2.27 & apf \\
\hline $2,458,732.0252$ & -7.23 & 2.32 & apf \\
\hline $2,458,733.1529$ & 0.70 & 1.04 & hires $_{j}$ \\
\hline $2,458,744.1572$ & -2.81 & 1.16 & hires $_{j}$ \\
\hline $2,458,746.8760$ & -2.41 & 2.33 & apf \\
\hline $2,458,746.8768$ & 3.22 & 2.40 & apf \\
\hline $2,458,746.8777$ & 1.49 & 2.39 & apf \\
\hline $2,458,746.8785$ & 2.54 & 2.26 & apf \\
\hline $2,458,747.8382$ & 6.14 & 2.72 & apf \\
\hline $2,458,747.8391$ & 0.56 & 2.66 & apf \\
\hline $2,458,747.8401$ & 2.39 & 2.84 & apf \\
\hline $2,458,747.8410$ & 5.68 & 2.69 & apf \\
\hline $2,458,749.8802$ & 1.21 & 2.64 & apf \\
\hline $2,458,749.8812$ & 5.31 & 2.52 & apf \\
\hline $2,458,749.8822$ & 3.23 & 2.64 & apf \\
\hline $2,458,752.9836$ & -15.60 & 2.07 & apf \\
\hline $2,458,752.9844$ & -11.82 & 2.24 & apf \\
\hline $2,458,752.9852$ & -10.04 & 2.22 & apf \\
\hline $2,458,752.9860$ & -12.86 & 2.27 & apf \\
\hline $2,458,765.8514$ & 0.59 & 2.51 & apf \\
\hline $2,458,765.8523$ & -1.54 & 2.25 & apf \\
\hline $2,458,765.8533$ & -5.55 & 2.65 & apf \\
\hline $2,458,776.9385$ & -2.09 & 1.14 & hires $_{j}$ \\
\hline $2,458,794.9139$ & 10.33 & 1.06 & $\operatorname{hires}_{j}$ \\
\hline $2,458,795.9719$ & 11.51 & 0.96 & hires $_{j}$ \\
\hline $2,458,796.9731$ & 8.51 & 1.17 & hires $_{j}$ \\
\hline $2,458,797.9759$ & 3.21 & 1.01 & hires $_{j}$ \\
\hline $2,458,798.8832$ & 15.73 & 3.20 & apf \\
\hline $2,458,798.8838$ & 15.91 & 4.46 & apf \\
\hline $2,458,798.8844$ & 10.47 & 5.65 & apf \\
\hline $2,458,798.9255$ & 9.65 & 1.05 & hires $_{j}$ \\
\hline $2,458,800.7426$ & 0.40 & 8.44 & apf \\
\hline $2,458,800.7431$ & -11.34 & 10.11 & apf \\
\hline $2,458,800.7437$ & 0.37 & 8.28 & apf \\
\hline $2,458,802.9074$ & -1.55 & 1.04 & hires $_{j}$ \\
\hline $2,458,819.9272$ & -1.62 & 1.23 & hires $_{j}$ \\
\hline $2,458,819.9278$ & 2.20 & 1.17 & hires $_{j}$ \\
\hline $2,458,819.9285$ & -1.86 & 1.25 & hires $_{j}$ \\
\hline $2,458,880.7768$ & 14.95 & 1.09 & hires $_{j}$ \\
\hline $2,458,905.7045$ & 7.52 & 0.93 & hires $_{j}$ \\
\hline $2,458,906.7048$ & 9.72 & 1.01 & hires $_{j}$ \\
\hline $2,458,907.7049$ & 11.37 & 1.05 & hires $_{j}$ \\
\hline $2,459,064.1408$ & 0.66 & 0.88 & hires $_{j}$ \\
\hline $2,459,067.1418$ & 4.26 & 0.92 & hires $_{j}$ \\
\hline $2,459,069.0914$ & 16.14 & 0.91 & $\operatorname{hires}_{j}$ \\
\hline $2,459,079.1461$ & 6.80 & 0.97 & hires $_{j}$ \\
\hline $2,459,088.1435$ & -0.35 & 0.83 & hires $_{j}$ \\
\hline $2,459,089.1488$ & -0.30 & 1.06 & hires $_{j}$ \\
\hline $2,459,090.1506$ & 5.99 & 0.85 & hires $_{j}$ \\
\hline $2,459,091.1516$ & 12.06 & 0.89 & hires $_{j}$ \\
\hline $2,459,117.1542$ & 16.88 & 1.01 & hires $_{j}$ \\
\hline $2,459,118.1551$ & 17.29 & 0.96 & hires $_{j}$ \\
\hline $2,459,120.0833$ & 16.54 & 0.94 & hires $_{j}$ \\
\hline
\end{tabular}

\section{Appendix B Hipparcos IAD Measurements}

The full list of IAD measurements of $\epsilon$ Eridani are shown in Table 6.

Table 6

Hipparcos IAD Measurements

\begin{tabular}{|c|c|c|}
\hline $\begin{array}{l}\text { Time } \\
(\mathrm{yr})\end{array}$ & $\begin{array}{c}\text { R.A. } \\
\left({ }^{\circ}\right)\end{array}$ & $\begin{array}{c}\text { Decl. } \\
\left({ }^{\circ}\right)\end{array}$ \\
\hline 1990.036 & 52.5116405005777 & -9.4583497858903 \\
\hline 1990.036 & 52.5116401584946 & -9.4583496322689 \\
\hline 1990.036 & 52.5116403587071 & -9.4583497221005 \\
\hline 1990.036 & 52.5116403865140 & -9.4583497347661 \\
\hline 1990.165 & 52.5115962411255 & -9.4583180708649 \\
\hline 1990.165 & 52.5115959467574 & -9.4583177566427 \\
\hline 1990.165 & 52.5115960096074 & -9.4583178233896 \\
\hline 1990.537 & 52.5116469916317 & -9.4582724641828 \\
\hline 1990.537 & 52.5116469841479 & -9.4582725080033 \\
\hline 1990.598 & 52.5116419591556 & -9.4582840685775 \\
\hline 1990.598 & 52.5116414487723 & -9.4582837747986 \\
\hline 1990.598 & 52.5116417640389 & -9.4582839565381 \\
\hline 1990.598 & 52.5116420312751 & -9.4582841103275 \\
\hline 1990.986 & 52.5114013929774 & -9.4583510281954 \\
\hline 1990.986 & 52.5114013393019 & -9.4583509989263 \\
\hline 1990.986 & 52.5114015402335 & -9.4583511001388 \\
\hline 1990.986 & 52.5114012050044 & -9.4583509320788 \\
\hline 1990.986 & 52.5114009365369 & -9.4583507920843 \\
\hline 1990.986 & 52.5114013955396 & -9.4583510292821 \\
\hline 1990.986 & 52.5114010690108 & -9.4583508624671 \\
\hline 1991.033 & 52.5113714176233 & -9.4583447128771 \\
\hline 1991.033 & 52.5113714135922 & -9.4583447403729 \\
\hline 1991.033 & 52.5113713999200 & -9.4583448169388 \\
\hline 1991.033 & 52.5113714029650 & -9.4583448033679 \\
\hline 1991.211 & 52.5113225169863 & -9.4582994758077 \\
\hline 1991.211 & 52.5113226736580 & -9.4582992298002 \\
\hline 1991.211 & 52.5113224771832 & -9.4582995327838 \\
\hline 1991.211 & 52.5113225766121 & -9.4582993787588 \\
\hline 1991.211 & 52.5113225353019 & -9.4582994446619 \\
\hline 1991.211 & 52.5113226173269 & -9.4582993157660 \\
\hline 1991.211 & 52.5113225487930 & -9.4582994236099 \\
\hline 1991.211 & 52.5113226206707 & -9.4582993113122 \\
\hline 1991.233 & 52.5113235973147 & -9.4582931095657 \\
\hline 1991.233 & 52.5113235887250 & -9.4582928458202 \\
\hline 1991.233 & 52.5113235967572 & -9.4582930845733 \\
\hline 1991.233 & 52.5113236047697 & -9.4582933483541 \\
\hline 1991.233 & 52.5113235890592 & -9.4582926401783 \\
\hline 1991.233 & 52.5113235894297 & -9.4582926262907 \\
\hline 1991.233 & 52.5113235941072 & -9.4582930012824 \\
\hline 1991.233 & 52.5113236004370 & -9.4582932122974 \\
\hline 1991.487 & 52.5113727458625 & -9.4582615280949 \\
\hline 1991.487 & 52.5113727288772 & -9.4582614578977 \\
\hline 1991.487 & 52.5113727022689 & -9.4582613500171 \\
\hline 1991.487 & 52.5113727581897 & -9.4582615765491 \\
\hline 1991.487 & 52.5113728058925 & -9.4582617679102 \\
\hline 1991.487 & 52.5113727896355 & -9.4582617118418 \\
\hline 1991.524 & 52.5113756856158 & -9.4582652690679 \\
\hline 1991.524 & 52.5113756602019 & -9.4582652578526 \\
\hline 1991.617 & 52.5113670292940 & -9.4582828689332 \\
\hline 1991.617 & 52.5113670158256 & -9.4582828932237 \\
\hline 1991.618 & 52.5113670300430 & -9.4582826870404 \\
\hline 1991.618 & 52.5113667689413 & -9.4582831584554 \\
\hline 1991.714 & 52.5113300285460 & -9.4583083881100 \\
\hline 1991.714 & 52.5113300175102 & -9.4583083656777 \\
\hline
\end{tabular}


Table 6

(Continued)

\begin{tabular}{lcc}
\hline $\begin{array}{l}\text { Time } \\
(\mathrm{yr})\end{array}$ & $\begin{array}{c}\text { R.A. } \\
\left({ }^{\circ}\right)\end{array}$ & $\begin{array}{c}\text { Decl. } \\
\left({ }^{\circ}\right)\end{array}$ \\
\hline 1991.714 & 52.5113301324919 & -9.4583086000961 \\
1991.714 & 52.5113300055516 & -9.4583083406066 \\
1991.714 & 52.5113299595752 & -9.4583082455852 \\
1991.714 & 52.5113300402794 & -9.4583084132933 \\
1991.715 & 52.5113293922848 & -9.4583084348328 \\
1991.715 & 52.5113293727876 & -9.4583083887744 \\
1991.715 & 52.5113294670004 & -9.4583085961528 \\
1992.062 & 52.5110857608807 & -9.4583343386895 \\
1992.062 & 52.5110853444093 & -9.4583341343475 \\
1992.062 & 52.5110851647440 & -9.4583340464981 \\
1992.062 & 52.5110856265134 & -9.4583342719861 \\
1992.062 & 52.5110853695640 & -9.4583341461406 \\
1992.139 & 52.5110586465189 & -9.4583148046891 \\
1992.139 & 52.5110585719244 & -9.4583149218441 \\
1992.139 & 52.5110585808650 & -9.4583149077813 \\
1992.139 & 52.5110585956311 & -9.4583148842530 \\
1992.189 & 52.5110520744159 & -9.4583002913271 \\
1992.189 & 52.5110521033931 & -9.4583003354390 \\
1992.189 & 52.5110521308103 & -9.4583003772504 \\
1992.189 & 52.5110519452936 & -9.4583000936515 \\
1992.563 & 52.5111043447049 & -9.4582660986126 \\
1992.563 & 52.5111044409491 & -9.4582658056901 \\
1992.563 & 52.5111043696635 & -9.4582660220212 \\
1992.563 & 52.5111043689974 & -9.4582660247248 \\
\hline & &
\end{tabular}

\section{Appendix C Using the Kernel Density Estimator}

The bandwidth is in practice associated with the smoothing of the kernel, and has to be carefully tuned to (1) avoid data artifacts caused by undersmoothing, and (2) retain the distribution tail information and skewed bounds limits that could be lost by oversmoothing. In Figure 8(a) we illustrate these effects and the importance of bandwidth selection. The value of the optimal bandwidth is, however, heavily dependent on the data; a higher amount of data points allows for a more aggressive bandwidth, i.e., lower bandwidth, and a low number of data points is more susceptible to a spurious ridged distribution.

A way of choosing the KDE bandwidth is the following. (1) Pick an acceptable change in the median and 68th interval limits of the KDE fit w.r.t the actual posterior distribution. This will set a maximum acceptable bandwidth. (2) Pick an acceptable variation of the log-prior probability when evaluating the priors for a SMA around the prior median SMA. This will set a minimum acceptable bandwidth. For this we will loop over a set of bandwidths computing the median and 68th interval limits, and for each bandwidth we will compute the variation of log-prior with a set of SMAs around the prior median SMA. We will fit a Gaussian and the standard deviation of the residuals to the fit will be our cost function. 

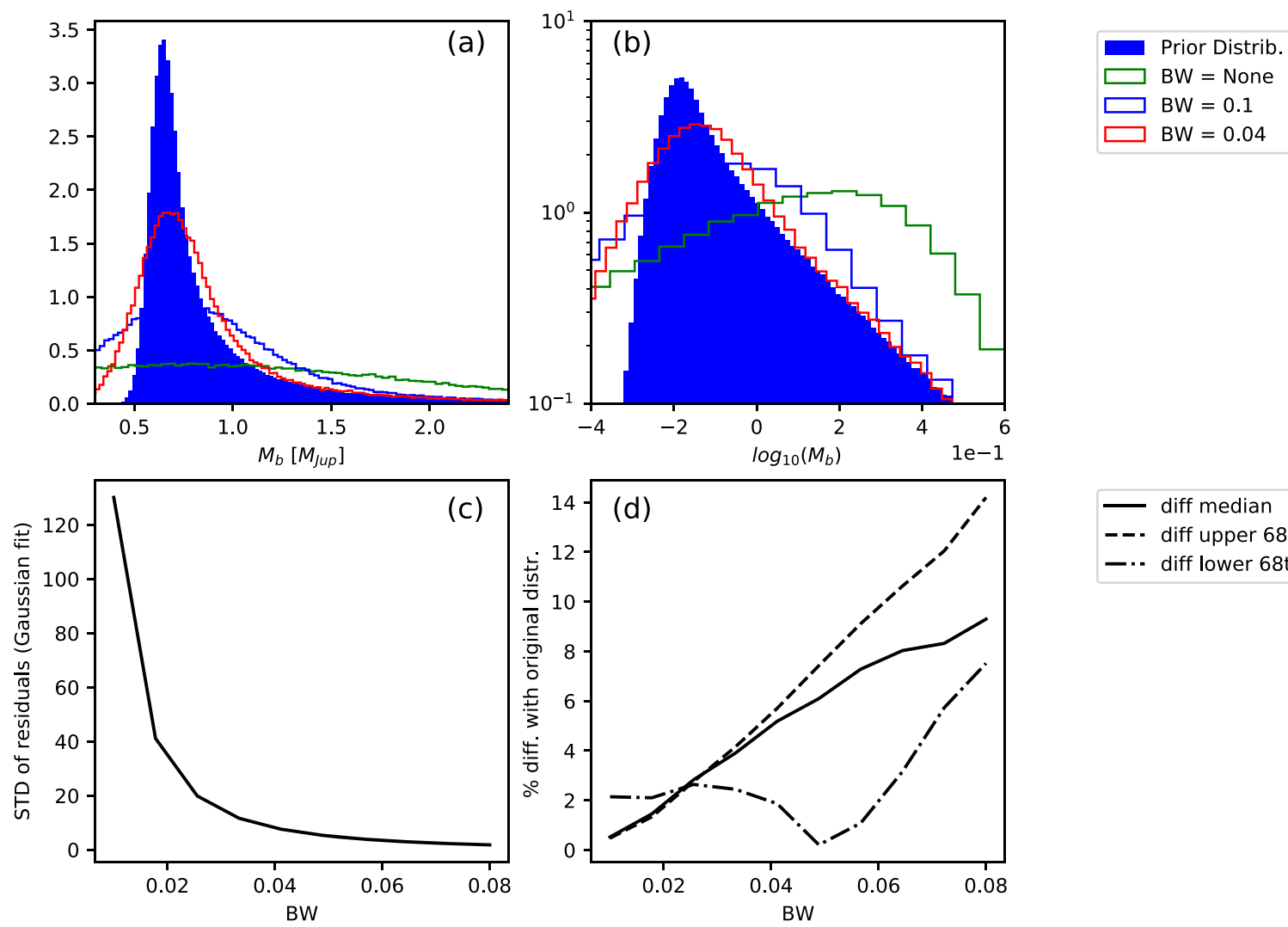

- diff median
--. diff upper 68th interval limit
-.. diff lower 68th interval limit

Figure 8. (a) Mass histogram comparing three different KDE bandwidths to fit the six-correlated parameters from the RV fit. The blue bar histogram represents the distribution from the RV fit, the step histograms are KDE fit to that distribution for different bandwidths. (b) The residuals in prior probability to a Gaussian fit versus the KDE bandwidth. For a set of reasonable SMAs, we compute the prior probabilities, and we fit a Gaussian; the narrower the bandwidth, the more the spurious effects of the RV posterior sampling affect the KDE fit. (c) Difference between the KDE fit and the original prior distribution for the mass distribution. The narrower the bandwidth, the closer to the original distribution. (d) Difference of the confidence intervals with respect to the original distribution. The narrower the bandwidth the more the upper and lower bounds are similar to the original distribution. A balance, thus, needs to be found between a small enough bandwidth so that the upper and lower bounds are well reproduced (see (d)), but not as small as to begin an undersmoothing effect (see (c)). 


\section{Appendix D}

\section{Perturbed Orbit Solution}

The perturbed orbit for the case of $800 \mathrm{Myr}$ is shown in Figure 9; the perturbation size is $\alpha_{A}=0.89$ mas. Overplotted are the estimated positions of $\epsilon$ Eridani for the Hipparcos and Gaia epochs; Hipparcos covers $\sim 35 \%$ of the orbit, Gaia epoch is taken as 2015.5 .

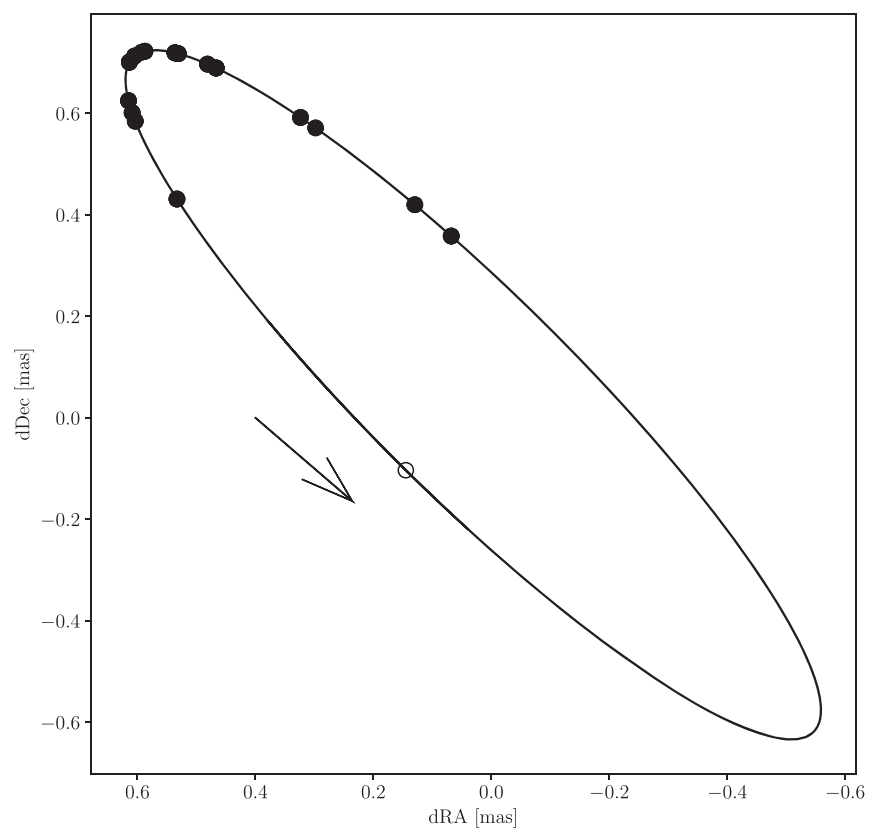

Figure 9. $\epsilon$ Eridani's perturbed orbit caused by the presence of the companion. Filled circles indicate the estimated position of the star at the Hipparcos IAD epochs, the empty circle indicates the Gaia DR2 epoch. The arrow indicates the direction of motion. 


\section{Appendix E \\ Corner Plot}

In Figure 10 the full corner plot is shown, with the posterior distributions and their correlation for the six orbital parameters and the masses. These posteriors correspond to the MCMC run for the fit to the RV, astrometry, and direct imaging data assuming an age of $800 \mathrm{Myr}$.

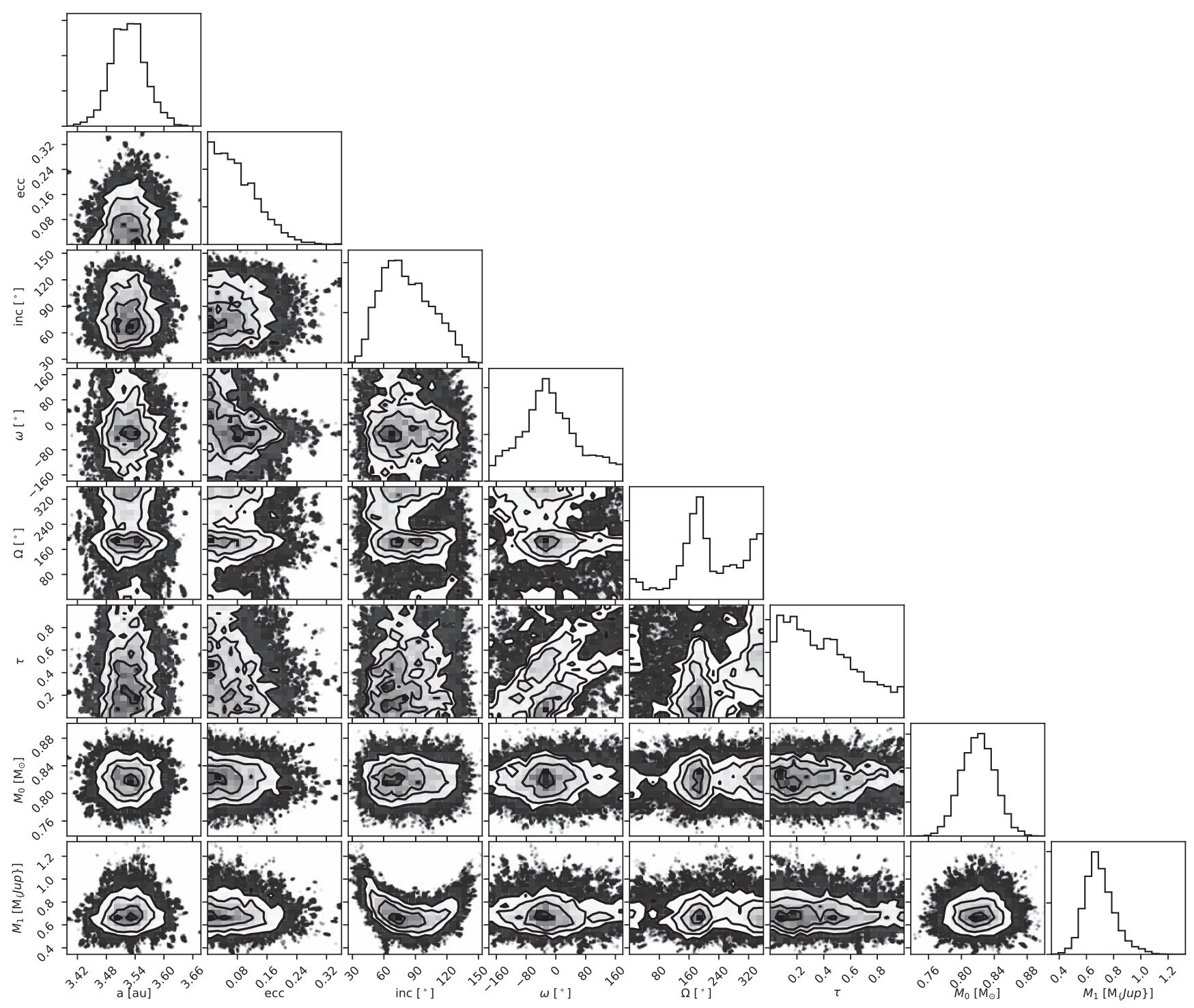

Figure 10. Posteriors of the orbital parameters and masses. 


\section{ORCID iDs}

Jorge Llop-Sayson (1) https://orcid.org/0000-0002-3414-784X Jason J. Wang (i) https://orcid.org/0000-0003-0774-6502 Dimitri Mawet (1) https://orcid.org/0000-0002-8895-4735 Sarah Blunt (ib https://orcid.org/0000-0002-3199-2888 Olivier Absil (i) https://orcid.org/0000-0002-4006-6237 Brendan P. Bowler (10) https://orcid.org/0000-0003-2649-2288 Ashley Chontos $\mathbb{1}$ ( https://orcid.org/0000-0003-1125-2564 Paul A. Dalba (1) https://orcid.org/0000-0002-4297-5506 B. J. Fulton (1i) https://orcid.org/0000-0003-3504-5316 Steven Giacalone (1) https://orcid.org/0000-0002-8965-3969 Lea A. Hirsch (1) https://orcid.org/0000-0001-8058-7443 Andrew W. Howard (1) https://orcid.org/0000-00018638-0320

Howard Isaacson (1) https://orcid.org/0000-0002-0531-1073 Mikael Karlsson (i) https://orcid.org/0000-0002-2011-0851 Jack Lubin (1) https://orcid.org/0000-0001-8342-7736 Marshall Perrin (1) https://orcid.org/0000-0002-3191-8151 Bin Ren (1) https://orcid.org/0000-0003-1698-9696 Malena Rice (i) https://orcid.org/0000-0002-7670-670X Lee J. Rosenthal (1) https://orcid.org/0000-0001-8391-5182 Ryan Rubenzahl (1) https://orcid.org/0000-0003-3856-3143 He Sun (1) https://orcid.org/0000-0003-1526-6787 Nicole Wallack (1) https://orcid.org/0000-0003-0354-0187

\section{References}

Astropy Collaboration, Robitaille, T. P., Tollerud, E. J., et al. 2013, A\&A, 558, A33

Aumann, H. H. 1985, PASP, 97, 885

Backman, D., Marengo, M., Stapelfeldt, K., et al. 2008, ApJ, 690, 1522

Baines, E. K., \& Armstrong, J. T. 2011, ApJ, 744, 138

Baraffe, I., Chabrier, G., Barman, T. S., Allard, F., \& Hauschildt, P. H. 2003, A\&A, 402, 701

Barman, T. S., Konopacky, Q. M., Macintosh, B., \& Marois, C. 2015, ApJ, 804,61

Beichman, C., Ygouf, M., Llop Sayson, J., et al. 2020, PASP, 132, 015002 Benedict, G. F., McArthur, B. E., Gatewood, G., et al. 2006, AJ, 132, 2206

Blunt, S., Endl, M., Weiss, L. M., et al. 2019, AJ, 158, 181

Blunt, S., Wang, J. J., Angelo, I., et al. 2020, AJ, 159, 89

Boccaletti, A., Lagage, P.-O., Baudoz, P., et al. 2015, PASP, 127, 633

Bond, C. Z., Cetre, S., Lilley, S., et al. 2020, JATIS, 6, 039003

Booth, M., Dent, W. R. F., Jordán, A., et al. 2017, MNRAS, 469, 3200

Brandl, B., Bettonvil, F., van Boekel, R., et al. 2021, Msngr, 182, 22

Brandt, T. D. 2021, ApJS, 254, 42

Carlomagno, B., Delacroix, C., Absil, O., et al. 2020, JATIS, 6, 035005

Carrión-González, Ó., García Muñoz, A., Cabrera, J., et al. 2021, A\&A, 651, A7

Cox, A. N. 2000, Allen's Astrophysical Quantities (New York: Springer)

Dawson, R. I., Murray-Clay, R. A., \& Fabrycky, D. C. 2011, ApJ, 743, L17

Ducati, J. R. 2002, yCat, 2002, 2237

Ertel, S., Defrère, D., Hinz, P., et al. 2018, AJ, 155, 194

Fitzgerald, M., Bailey, V., Baranec, C., et al. 2019, BAAS, 51, 251

Foreman-Mackey, D. 2016, JOSS, 1, 24

Foreman-Mackey, D., Hogg, D. W., Lang, D., \& Goodman, J. 2013, PASP, 125,306

Fulton, B. J., Petigura, E. A., Blunt, S., \& Sinukoff, E. 2018, PASP, 130, 044504
Gaia Collaboration, Brown, A. G. A., Vallenari, A., et al. 2018, A\&A, 616, A1 GRAVITY Collaboration, Nowak, M., Lacour, S., et al. 2020, A\&A, 633, A110

Hatzes, A. P., Cochran, W. D., McArthur, B., et al. 2000, ApJL, 544, L145

Hinkley, S., Matthews, E. C., Lefevre, C., et al. 2021, ApJ, 912, 115

Hoeijmakers, H. J., Schwarz, H., Snellen, I. A. G., et al. 2018, A\&A, 617, A144

Hou, F., Goodman, J., Hogg, D. W., Weare, J., \& Schwab, C. 2012, ApJ, 745,198

Houllé, M., Vigan, A., Carlotti, A., et al. 2021, A\&A, 652, A67

Howard, A. W., Johnson, J. A., Marcy, G. W., et al. 2010, ApJ, 721, 1467

Huby, E., Baudoz, P., Mawet, D., \& Absil, O. 2015, A\&A, 584, A74

Huby, E., Bottom, M., Femenia, B., et al. 2017, A\&A, 600, A46

Hunter, J. D. 2007, CSE, 9, 90

Janson, M., Quanz, S. P., Carson, J. C., et al. 2015, A\&A, 574, A120

Jones, E., Oliphant, T., \& Peterson, P. 2001, SciPy: Open Source Scientific Tools for Python

Kasper, M., Arsenault, R., Käufl, U., et al. 2019, Msngr, 178, 5

Keenan, P. C., \& McNeil, R. C. 1989, ApJS, 71, 245

Kervella, P., Arenou, Frédéric, Mignard, François, \& Thévenin, Frédéric 2019, A\&A, 623, A72

Konopacky, Q. M., Barman, T. S., Macintosh, B. A., \& Marois, C. 2013, Sci, 339, 1398

Lindegren, L., Hernández, J., Bombrun, A., et al. 2018, A\&A, 616, A2

López-Morales, M., Haywood, R. D., Coughlin, J. L., et al. 2016, AJ, 152, 204

MacGregor, M. A., Wilner, D. J., Andrews, S. M., Lestrade, J.-F., \& Maddison, S. 2015, ApJ, 809, 47

Mamajek, E. E., \& Hillenbrand, L. A. 2008, ApJ, 687, 1264

Marley, M., Saumon, D., Morley, C., \& Fortney, J. 2018, Sonora 2018: Cloudfree, solar composition, solar C/O substellar atmosphere models and spectra, nc_m+0.0_co1.0_v1.0, Zenodo, doi:10.5281/zenodo.1309035

Marois, C., Lafrenière, D., Macintosh, B., \& Doyon, R. 2008, ApJ, 673, 647

Mawet, D., Hirsch, L., Lee, E. J., et al. 2019, AJ, 157, 33

Nielsen, E. L., De Rosa, R. J., Wang, J. J., et al. 2020, AJ, 159, 71

Nowak, M., Lacour, S., Lagrange, A. M., et al. 2020, A\&A, 642, L2

Pathak, P., Petit dit de la Roche, D. J. M., Kasper, M., et al. 2021, A\&A, 652, A121

Perrin, M. D., Pueyo, L., Gorkom, K. V., et al. 2018, Proc. SPIE, 10698, 98

Petit dit de la Roche, D. J. M., Hoeijmakers, H. J., \& Snellen, I. A. G. 2018 , A\&A, 616, A146

Quillen, A. C., \& Faber, P. 2006, MNRAS, 373, 1245

Radovan, M. V., Lanclos, K., Holden, B. P., et al. 2014, Proc. SPIE, 9145 , 91452B

Rajpaul, V., Aigrain, S., Osborne, M. A., Reece, S., \& Roberts, S. 2015, MNRAS, 452, 2269

Rosa, R. J. D., Esposito, T. M., Hirsch, L. A., et al. 2019a, AJ, 158, 225

Rosa, R. J. D., Nielsen, E. L., Wang, J. J., et al. 2019b, AJ, 159, 1

Rosenthal, Lee J., et al. 2021, ApJS, 255, 8

Ruffio, J.-B., Macintosh, B., Konopacky, Q. M., et al. 2019, AJ, 158, 200

Ruffio, J.-B., Macintosh, B., Wang, J. J., et al. 2017, ApJ, 842, 14

Ruffio, J.-B., Mawet, D., Czekala, I., et al. 2018, AJ, 156, 196

Serabyn, E., Huby, E., Matthews, K., et al. 2017, AJ, 153, 43

Soummer, R., Pueyo, L., \& Larkin, J. 2012, ApJ, 755, L28

Su, K. Y. L., De Buizer, J. M., Rieke, G. H., et al. 2017, AJ, 153, 226

van Leeuwen, F. 2007, Hipparcos, the New Reduction of the Raw Data, Vol. 350 (Berlin: Springer)

Vogt, S. S., Allen, S. L., Bigelow, B. C., et al. 1994, Proc. SPIE, 2198, 362

Vogt, S. S., Radovan, M., Kibrick, R., et al. 2014, PASP, 126, 359

Wang, J. J., Ruffio, J.-B., De Rosa, R. J., et al. 2015, pyKLIP: PSF Subtraction for Exoplanets and Disks, Astrophysics Source Code Library, ascl:1506.001

Wang, J. J., Vigan, A., Lacour, S., et al. 2021, AJ, 161, 148

Wilcomb, K. K., Konopacky, Q. M., Barman, T. S., et al. 2020, AJ, 160, 207

Wisdom, J. 1980, AJ, 85, 1122

Xuan, W. J., Mawet, D., Ngo, H., et al. 2018, AJ, 156, 156 